\title{
Preparation of stannyl complexes of ruthenium and osmium stabilised by polypyridine and phosphite ligands $\uparrow$
}

\author{
Gabriele Albertin, ${ }^{* a}$ Stefano Antoniutti, ${ }^{a}$ Jesús Castro, ${ }^{b}$ Soledad García-Fontán ${ }^{b}$ and Marco Noé ${ }^{a}$ \\ Received 23rd May 2007, Accepted 17th September 2007 \\ First published as an Advance Article on the web 4th October 2007 \\ DOI: $10.1039 /$ b707736j
}

Trichlorostannyl complexes $\left[\mathrm{M}\left(\mathrm{SnCl}_{3}\right)(\mathrm{bpy})_{2} \mathrm{P}\right] \mathrm{BPh}_{4}\left[\mathrm{M}=\mathrm{Ru}, \mathrm{P}=\mathrm{P}(\mathrm{OEt})_{3} \mathbf{1 a}, \mathrm{PPh}(\mathrm{OEt})_{2} \mathbf{1 b} ; \mathrm{M}=\mathrm{Os}\right.$, $\mathrm{P}=\mathrm{P}(\mathrm{OEt})_{3} 2$; bpy $=2,2^{\prime}$-bipyridine $]$ were prepared by allowing chloro complexes $\left[\mathrm{MCl}(\mathrm{bpy})_{2} \mathrm{P}_{\mathrm{BPh}} \mathrm{BP}_{4}\right.$ to react with $\mathrm{SnCl}_{2}$ in 1,2-dichloroethane. Bis(trichlorostannyl) compounds $\mathrm{Ru}\left(\mathrm{SnCl}_{3}\right)_{2}(\mathrm{~N}-\mathrm{N}) \mathrm{P}_{2}$ $\left[\mathrm{N}-\mathrm{N}=\right.$ bpy, $\mathrm{P}=\mathrm{P}(\mathrm{OEt})_{3}$ 3a, $\mathrm{PPh}(\mathrm{OEt})_{2} 3 \mathbf{b} ; \mathrm{N}-\mathrm{N}=1,10$-phenanthroline (phen), $\left.\mathrm{P}=\mathrm{P}(\mathrm{OEt})_{3} 4\right]$ were also prepared by reacting $\left[\mathrm{RuCl}(\mathrm{N}-\mathrm{N}) \mathrm{P}_{3}\right] \mathrm{BPh}_{4}$ precursors with $\mathrm{SnCl}_{2} \cdot 2 \mathrm{H}_{2} \mathrm{O}$ in ethanol. Treatment of both mono- 1a, 2 and bis 3a trichlorostannyl complexes with $\mathrm{NaBH}_{4}$ afforded mono- and bis(trihydridestannyl) derivatives $\left[\mathrm{M}\left(\mathrm{SnH}_{3}\right)(\mathrm{bpy})_{2} \mathrm{P} \mathrm{BPh}_{4} \mathbf{5}, \mathbf{6}\right.$ and $\mathrm{Ru}\left(\mathrm{SnH}_{3}\right)_{2}($ bpy $) \mathrm{P}_{2} 7\left[\mathrm{P}=\mathrm{P}(\mathrm{OEt})_{3}\right]$, respectively. Treatment of 1a, $\mathbf{2}$ with $\mathrm{MgBrMe}$ gave the trimethylstannyl complexes $\left[\mathrm{M}\left(\mathrm{SnMe}_{3}\right)(\mathrm{bpy})_{2} \mathrm{P}_{3} \mathrm{BPh}_{4} \mathbf{8}, \mathbf{9}\right.$, and treatment of $\mathbf{3 a}$ afforded the bis(stannyl) $\mathrm{Ru}\left(\mathrm{SnClMe}_{2}\right)_{2}(\mathrm{bpy}) \mathrm{P}_{2} \mathbf{1 0}$ derivative. Alkynylstannyl complexes $\left[\mathrm{M}\left\{\mathrm{Sn}(\mathrm{C} \equiv \mathrm{CR})_{3}\right\}(\text { bpy })_{2} \mathrm{P}\right] \mathrm{BPh}_{4} \mathbf{1 1}-\mathbf{1 3}$ and $\mathrm{Ru}\left[\mathrm{Sn}(\mathrm{C} \equiv \mathrm{CR})_{3}\right]_{2}(\mathrm{~N}-\mathrm{N}) \mathrm{P}_{2}$ 14-17 $\left(\mathrm{R}=p\right.$-tolyl, $\mathrm{Bu}^{t} ; \mathrm{N}-\mathrm{N}=$ bpy, phen) were also prepared by allowing trichlorostannyl compounds 1-4 to react with $\mathrm{Li}^{+}\left[\mathrm{RC} \equiv \mathrm{C}^{-}\right.$in thf. The complexes were characterised spectroscopically and by the X-ray crystal structure determination of $\left[\mathrm{Ru}\left(\mathrm{SnMe}_{3}\right)(\mathrm{bpy})_{2}\left\{\mathrm{P}(\mathrm{OEt})_{3}\right\}\right] \mathrm{BPh}_{4} \mathbf{8}$ derivative.

\section{Introduction}

Transition-metal stannyl complexes [M]-SnX ${ }_{3}$ and [M]-SnR ${ }_{3}$ have been extensively studied in the past 25 years, ${ }^{1-4}$ because of the variety of reactions that they may undergo, including ligand substitution to the metal centre and nucleophilic reactions at the stannyl group. Tin ligands are also widely used to modify the activity of noble metal catalysts. ${ }^{1,4}$

Among ligands used in transition-metal stannyl chemistry, a prominent role is played by $\pi$-acceptors such as $\mathrm{CO}$, tertiary phosphine, and cyclopentadienyls. ${ }^{1-3}$ Less attention has been paid to nitrogen donors as ancillary ligands, and only a few examples of stannyl complexes stabilised by $\alpha$-diimine or tetraazabutadiene ligands have been reported so far. ${ }^{5}$

We are interested in the synthesis and reactivity of transition metal complexes containing nitrogen donors and phosphites as ancillary ligands, and have recently reported some results on polypyridine $\left[\mathrm{MX}(\mathrm{N}-\mathrm{N}) \mathrm{P}_{3}\right]^{+},\left[\mathrm{MX}(\mathrm{N}-\mathrm{N})_{2} \mathrm{P}\right]^{+}\left(\mathrm{N}-\mathrm{N}=2,2^{\prime}-\right.$ bipyridine, 1,10-phenanthroline) and tris(pyrazolyl)borate (Tp) $\left[\mathrm{MX}(\mathrm{Tp}) \mathrm{L}_{2}\right]^{+}(\mathrm{X}=\mathrm{Cl}, \mathrm{H})$ complexes of the iron triad. ${ }^{6,7} \mathrm{We}$ also observed ${ }^{8}$ that the use of the tris(pyrazolyl)borate ligand in mixed-ligand ruthenium and osmium complexes gave rise to the synthesis of the first complexes containing tin trihydride [M]- $\mathrm{SnH}_{3}$ as a ligand. We have now extended these studies, with the aim of verifying whether 2,2'-bipyridine (bpy) and 1,10-phenanthroline (phen) as supporting ligands can give rise to the synthesis of

${ }^{a}$ Dipartimento di Chimica, Università Ca' Foscari di Venezia, Dorsoduro, 2137,30123Venezia, Italy.E-mail:albertin@unive.it

${ }^{b}$ Departamento de Química Inorgánica, Universidade de Vigo, Facultade de Quimica, Edificio de Ciencias Experimentais, 36310 Vigo (Galicia), Spain $\dagger$ CCDC reference numbers 648279. For crystallographic data in CIF or other electronic format see DOI: 10.1039/b707736j stable tin trihydride complexes. The results of these studies, which involve the preparation of novel mono and bis(stannyl) complexes of ruthenium and osmium, are reported here.

\section{Experimental}

\section{General comments}

All synthetic work was carried out in an appropriate atmosphere $\left(\mathrm{Ar}, \mathrm{N}_{2}\right.$ ), by means of standard Schlenk techniques or a vacuum atmosphere dry-box. Once isolated, the complexes were found to be stable in air and were handled without particular caution. All solvents were dried over appropriate drying agents, degassed on a vacuum line, and distilled into vacuum-tight storage flasks. $\mathrm{RuCl}_{3} \cdot 3 \mathrm{H}_{2} \mathrm{O}$ and $\left(\mathrm{NH}_{4}\right)_{2} \mathrm{OsCl}_{6}$ salts were Pressure Chemical Co. (USA) products, used as received. The phosphite $\mathrm{PPh}(\mathrm{OEt})_{2}$ was prepared by the method of Rabinowitz and Pellon. ${ }^{9}$ The reagent $\mathrm{MgBrMe}\left(3 \mathrm{~mol} \mathrm{dm}^{-3}\right.$ solution in diethylether) was an Aldrich product used as received. Lithium acetylide $\mathrm{Li}^{+}\left[\mathrm{RC} \equiv \mathrm{C}^{-}(\mathrm{R}=p\right.$ tolyl) was prepared by reacting a slight excess of the appropriate acetylene $(35 \mathrm{mmol})$ with lithium $(30 \mathrm{mmol}, 0.21 \mathrm{~g})$ in $20 \mathrm{~cm}^{3}$ of tetrahydrofuran (thf). tert-Butyl acetylide $\mathrm{Li}^{+}\left[\mathrm{Bu}^{t} \mathrm{C} \equiv \mathrm{C}^{-}\right.$was prepared by adding a solution of $\mathrm{LiBu}^{n} 2.5 \mathrm{~mol} \mathrm{dm}^{-3}$ in hexane $\left(15 \mathrm{mmol}, 6.0 \mathrm{~cm}^{3}\right)$ to a solution of $\mathrm{Bu}^{t} \mathrm{C} \equiv \mathrm{CH}\left(20 \mathrm{mmol}, 2.46 \mathrm{~cm}^{3}\right)$ in $10 \mathrm{~cm}^{3}$ of thf cooled to $-80^{\circ} \mathrm{C}$. The reaction mixture was stirred for $20-30 \mathrm{~min}$ at $-80{ }^{\circ} \mathrm{C}$ and then used. Other reagents were purchased from commercial sources in the highest available purity and used as received. Infrared spectra were recorded on a PerkinElmer Spectrum One FT-IR spectrophotometer. NMR spectra $\left({ }^{1} \mathrm{H},{ }^{31} \mathrm{P},{ }^{13} \mathrm{C},{ }^{119} \mathrm{Sn}\right)$ were obtained on AC200 or AVANCE 300 Bruker spectrometers at temperatures between -90 and $+30{ }^{\circ} \mathrm{C}$, unless otherwise noted. ${ }^{1} \mathrm{H}$ and ${ }^{13} \mathrm{C}$ spectra are referred to internal 
tetramethylsilane; ${ }^{31} \mathrm{P}\left\{{ }^{1} \mathrm{H}\right\}$ chemical shifts are reported with respect to $85 \% \mathrm{H}_{3} \mathrm{PO}_{4}$ and those of ${ }^{119} \mathrm{Sn}$ with respect to $\mathrm{Sn}\left(\mathrm{CH}_{3}\right)_{4}$ and, in both cases, downfield shifts were considered positive. COSY, HMQC and HMBC NMR experiments were performed with standard programs. The SwaN-MR and iNMR software packages ${ }^{10}$ were used to treat NMR data. The conductivity of $10^{-3}$ solutions of the complexes in $\mathrm{CH}_{3} \mathrm{NO}_{2}$ (or acetone) at $25{ }^{\circ} \mathrm{C}$ were measured on a Radiometer CDM 83. Elemental analyses were determined in the Microanalytical Laboratory of the Dipartimento di Scienze Farmaceutiche, University of Padova (Italy).

\section{Synthesis of complexes}

$\left[\mathrm{MCl}(\text { bpy })_{2} \mathrm{P}\right] \mathrm{BPh}_{4}[\mathrm{M}=\mathrm{Ru}, \mathrm{Os} ;$ bpy $=1$,2-bipyridine; $\mathrm{P}=$ $\left.\mathrm{P}(\mathrm{OEt})_{3}, \mathrm{PPh}(\mathrm{OEt})_{2}\right]$ and $\left[\mathrm{RuCl}(\mathrm{N}-\mathrm{N}) \mathrm{P}_{3}\right] \mathrm{BPh}_{4}[\mathrm{~N}-\mathrm{N}=$ bpy, 1,10phenanthroline (phen); $\left.\mathrm{P}=\mathrm{P}(\mathrm{OEt})_{3}, \mathrm{PPh}(\mathrm{OEt})_{2}\right]$ complexes were prepared following previously reported methods. ${ }^{6}$

\section{$\left[\mathrm{Ru}\left(\mathrm{SnCl}_{3}\right)(\mathrm{bpy})_{2} \mathrm{P}\right] \mathrm{BPh}{ }_{4} 1\left[\mathrm{P}=\mathrm{P}(\mathrm{OEt})_{3} 1 \mathrm{a}, \mathrm{PPh}(\mathrm{OEt})_{2}\right.$ 1b]}

Method A. In a $250-\mathrm{cm}^{3}$ three-necked round-bottomed flask were placed $1.0 \mathrm{mmol}$ of $\left[\mathrm{RuCl}(\mathrm{bpy})_{2} \mathrm{P}\right] \mathrm{BPh}_{4}, 5.0 \mathrm{mmol}(1.1 \mathrm{~g})$ of $\mathrm{SnCl}_{2} \cdot 2 \mathrm{H}_{2} \mathrm{O}$, and $80 \mathrm{~cm}^{3}$ of ethanol. The reaction mixture was refluxed for $4 \mathrm{~h}$ and then stirred at room temperature until a yellow gummy material separated out, which was collected and triturated with ethanol $\left(50 \mathrm{~cm}^{3}\right)$ containing an excess of $\mathrm{NaBPh}_{4}$ ( $2 \mathrm{mmol}, 0.68 \mathrm{~g}$ ). After $4 \mathrm{~h}$ of stirring, the orange-yellow solid which separated out was filtered and crystallised from $\mathrm{CH}_{2} \mathrm{Cl}_{2}$ and ethanol; yield $\geq 50 \%$.

Method B. In a $100-\mathrm{cm}^{3}$ three-necked round-bottomed flask were placed $2 \mathrm{mmol}$ of $\left[\mathrm{RuCl}(\text { bpy })_{2} \mathrm{P}\right] \mathrm{BPh}_{4}, 10.0 \mathrm{mmol}(1.9 \mathrm{~g})$ of anhydrous $\mathrm{SnCl}_{2}$, and $60 \mathrm{~cm}^{3}$ of dichloroethane. The reaction mixture was refluxed for $20 \mathrm{~min}$, cooled to room temperature and filtered to remove the unreacted $\mathrm{SnCl}_{2}$. The resulting solution was evaporated to dryness under reduced pressure to give an oil which was treated with ethanol $\left(20 \mathrm{~cm}^{3}\right)$ containing an excess of $\mathrm{NaBPh}_{4}$ (4 mmol, $1.37 \mathrm{~g}$ ). A yellow solid slowly separated out, which was filtered and crystallised from $\mathrm{CH}_{2} \mathrm{Cl}_{2}$ and ethanol; yield $\geq 90 \%$. (1a: ${ }^{1} \mathrm{H}$ NMR $\left(\mathrm{CD}_{2} \mathrm{Cl}_{2}, 20{ }^{\circ} \mathrm{C}\right) \delta$ : 9.41-6.75 (m, 36H, $\mathrm{Ph}+$ bpy), 3.87 (qnt, 6H, $\mathrm{CH}_{2}$ ), 1.09 (t, 9H, $\mathrm{CH}_{3}, J_{\mathrm{HH}}=7 \mathrm{~Hz}$ ). ${ }^{31} \mathrm{P}\left\{{ }^{1} \mathrm{H}\right\} \mathrm{NMR}$ $\left(\mathrm{CD}_{2} \mathrm{Cl}_{2}, 20^{\circ} \mathrm{C}\right) \delta$ : A spin system, $\delta_{\mathrm{A}} 129.6\left(J^{31} 1_{\mathrm{P}}{ }^{117} \mathrm{Sn}=523.4 \mathrm{~Hz}\right)$. $\Lambda_{\mathrm{M}} / \mathrm{S} \mathrm{cm} \mathrm{mol}^{-1}=53.3$. Found: $\mathrm{C}, 53.26 ; \mathrm{H}, 4.68 ; \mathrm{N}, 4.92 ; \mathrm{Cl}$, 9.24. $\mathrm{C}_{50} \mathrm{H}_{51} \mathrm{BCl}_{3} \mathrm{~N}_{4} \mathrm{O}_{3}$ PRuSn (1123.88) requires $\mathrm{C}$, 53.44; $\mathrm{H}, 4.57$; $\mathrm{N}, 4.99 ; \mathrm{Cl}, 9.46 \%$. 1b: ${ }^{1} \mathrm{H} \mathrm{NMR}\left(\mathrm{CD}_{2} \mathrm{Cl}_{2}, 20{ }^{\circ} \mathrm{C}\right) \delta: 9.70-6.69(\mathrm{~m}$, $41 \mathrm{H}, \mathrm{Ph}+\mathrm{bpy}), 4.18\left(\mathrm{~m}, 4 \mathrm{H}, \mathrm{CH}_{2}\right), 1.46,1.32\left(\mathrm{t}, 6 \mathrm{H}, \mathrm{CH}_{3}, J_{\mathrm{HH}}=\right.$ 7). ${ }^{31} \mathrm{P}\left\{{ }^{1} \mathrm{H}\right\}$ NMR $\left(\mathrm{CD}_{2} \mathrm{Cl}_{2}, 20{ }^{\circ} \mathrm{C}\right) \delta$ : A, $160.9\left(J^{31} \mathrm{p}_{\mathrm{P}}^{117} \mathrm{Sn}=330\right)$. $\Lambda_{\mathrm{M}} / \mathrm{S} \mathrm{cm} \mathrm{mol}^{-1}=54.1$. Found: C, 55.97; H, 4.38; N, 4.92; Cl, 9.05. $\mathrm{C}_{54} \mathrm{H}_{51} \mathrm{BCl}_{3} \mathrm{~N}_{4} \mathrm{O}_{2}$ PRuSn (1155.93) requires $\mathrm{C}, 56.11 ; \mathrm{H}, 4.45$; $\mathrm{N}, 4.85 ; \mathrm{Cl}, 9.20 \%$.)

\section{$\left[\mathrm{Os}\left(\mathrm{SnCl}_{3}\right)(\mathrm{bpy})_{2}\left\{\mathrm{P}(\mathrm{OEt})_{3}\right\}\right] \mathrm{BPh}_{4} 2$}

In a $100-\mathrm{cm}^{3}$ three-necked round-bottomed flask were placed $0.90 \mathrm{~g}(0.88 \mathrm{mmol})$ of $\left[\mathrm{OsCl}(\mathrm{bpy})_{2}\left\{\mathrm{P}(\mathrm{OEt})_{3}\right\}\right] \mathrm{BPh}_{4}, 1.0 \mathrm{~g}$ (5.3 mmol) of anhydrous $\mathrm{SnCl}_{2}$, and $40 \mathrm{~cm}^{3}$ of dichloroethane. The reaction mixture was refluxed for $1 \mathrm{~h}$, cooled to room temperature and filtered to remove the unreacted $\mathrm{SnCl}_{2}$. The resulting solution was concentrated to about $3 \mathrm{~cm}^{3}$ by evaporation under reduced pressure and then an excess of $\mathrm{NaBPh}_{4}(4 \mathrm{mmol}, 1.37 \mathrm{~g})$ in ethanol $\left(10 \mathrm{~cm}^{3}\right)$ was added. A brown solid slowly separated out from the stirring solution, which was filtered and crystallised from dichloroethane and ethanol; yield $\geq 90 \%$. ( ${ }^{1} \mathrm{H} \mathrm{NMR}\left(\mathrm{CD}_{2} \mathrm{Cl}_{2}\right.$, $\left.20{ }^{\circ} \mathrm{C}\right) \delta: 9.58-6.75(\mathrm{~m}, 36 \mathrm{H}, \mathrm{Ph}+\mathrm{bpy}), 3.81$ (qnt, 6H, $\mathrm{CH}_{2}$ ), $1.06\left(\mathrm{t}, 9 \mathrm{H}, \mathrm{CH}_{3}, J_{\mathrm{HH}}=7\right) .{ }^{31} \mathrm{P}\left\{{ }^{1} \mathrm{H}\right\} \operatorname{NMR}\left(\mathrm{CD}_{2} \mathrm{Cl}_{2}, 20{ }^{\circ} \mathrm{C}\right) \delta$ :

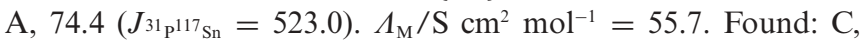
49.43; H, 4.18; N, 4.70; Cl, 8.64. $\mathrm{C}_{50} \mathrm{H}_{51} \mathrm{BCl}_{3} \mathrm{~N}_{4} \mathrm{O}_{3} \mathrm{OsPSn}$ (1213.01) requires $\mathrm{C}, 49.51 ; \mathrm{H}, 4.24 ; \mathrm{N}, 4.62 ; \mathrm{Cl}, 8.77 \%$.)

\section{$\mathrm{Ru}\left(\mathrm{SnCl}_{3}\right)_{2}(\mathrm{~N}-\mathrm{N}) \mathrm{P}_{2} 3,4\left[\mathrm{~N}-\mathrm{N}=\mathrm{bpy}, \mathrm{P}=\mathrm{P}(\mathrm{OEt})_{3} 3 \mathrm{a}\right.$, $\mathrm{PPh}(\mathrm{OEt})_{2} 3 \mathrm{~b} ; \mathrm{N}-\mathrm{N}=$ phen, $\left.\mathrm{P}=\mathrm{P}(\mathrm{OEt})_{3} 4\right]$}

In a $250-\mathrm{cm}^{3}$ three-necked round-bottomed flask were placed $0.9 \mathrm{mmol}$ of the appropriate $\left.\left[\mathrm{RuCl}(\mathrm{N}-\mathrm{N}) \mathrm{P}_{3}\right\}\right] \mathrm{BPh}_{4}$ complex, $9 \mathrm{mmol}(2 \mathrm{~g})$ of $\mathrm{SnCl}_{2} \cdot 2 \mathrm{H}_{2} \mathrm{O}$, and $80 \mathrm{~cm}^{3}$ of ethanol. The reaction mixture was refluxed for $4 \mathrm{~h}$ and then left to reach room temperature. A yellow solid slowly separated out from the resulting solution, which was filtered and crystallised from $\mathrm{CH}_{2} \mathrm{Cl}_{2}$ and ethanol; yield $\geq 60 \%$. (3a: ${ }^{1} \mathrm{H}$ NMR $\left(\mathrm{CD}_{2} \mathrm{Cl}_{2}, 20{ }^{\circ} \mathrm{C}\right)$ $\delta$ : 9.37-7.55 (m, 8H, bpy), $4.25\left(\mathrm{~m}, 12 \mathrm{H}, \mathrm{CH}_{2}\right), 1.44(\mathrm{t}, 18 \mathrm{H}$, $\left.\mathrm{CH}_{3}, J_{\mathrm{HH}}=7\right) .{ }^{31} \mathrm{P}\left\{{ }^{1} \mathrm{H}\right\} \operatorname{NMR}\left(\mathrm{CD}_{2} \mathrm{Cl}_{2}, 20{ }^{\circ} \mathrm{C}\right) \delta: \mathrm{A}_{2}, 138.1$ $\left(J^{31}{ }_{\mathrm{P} 117 \mathrm{Sn}}=373.7\right)$. Found: C, 25.55; H, 3.61; N, 2.77; Cl, 20.32. $\mathrm{C}_{22} \mathrm{H}_{38} \mathrm{Cl}_{6} \mathrm{~N}_{2} \mathrm{O}_{6} \mathrm{P}_{2} \mathrm{RuSn}_{2}$ (1039.67) requires $\mathrm{C}, 25.42 ; \mathrm{H}, 3.68 ; \mathrm{N}$, 2.69; $\mathrm{Cl}, 20.46 \%$. 3b: ${ }^{1} \mathrm{H}$ NMR $\left(\mathrm{CD}_{2} \mathrm{Cl}_{2}, 20{ }^{\circ} \mathrm{C}\right) \delta: 9.60-6.90(\mathrm{~m}$, $18 \mathrm{H}, \mathrm{Ph}+$ bpy), 4.45-4.02 (m, 8H, $\left.\mathrm{CH}_{2}\right), 1.47(\mathrm{~m}), 1.29(\mathrm{t})(12 \mathrm{H}$, $\left.\mathrm{CH}_{3}, J_{\mathrm{HH}}=7\right) .{ }^{31} \mathrm{P}\left\{{ }^{1} \mathrm{H}\right\} \mathrm{NMR}\left(\mathrm{CD}_{2} \mathrm{Cl}_{2}, 20{ }^{\circ} \mathrm{C}\right) \delta: \mathrm{AB}, \delta_{\mathrm{A}} 166.1, \delta_{\mathrm{B}}$ $154.4\left(J_{\mathrm{AB}}=47.6, J_{\mathrm{PA}}^{117} \mathrm{Sn}=344.0, J_{\mathrm{PB}^{117} \mathrm{Sn}}=455.8, J_{\mathrm{PA}^{117} \mathrm{Sn}}=332.4\right.$, $\left.J_{\mathrm{PB}}^{117_{\mathrm{Sn}}}=3043.6\right)$. Found: C, 32.51; H, 3.57; N, 2.48; Cl, 19.39. $\mathrm{C}_{30} \mathrm{H}_{38} \mathrm{Cl}_{6} \mathrm{~N}_{2} \mathrm{O}_{4} \mathrm{P}_{2} \mathrm{RuSn}_{2}$ (1103.76) requires C, 32.65; H, 3.47; N, 2.54; $\mathrm{Cl}, 19.27 \% .4:{ }^{1} \mathrm{H} \mathrm{NMR}\left(\mathrm{CD}_{2} \mathrm{Cl}_{2}, 20{ }^{\circ} \mathrm{C}\right) \delta: 9.73-7.64(\mathrm{~m}, 8 \mathrm{H}$, phen), $4.30\left(\mathrm{~m}, 12 \mathrm{H}, \mathrm{CH}_{2}\right), 1.47\left(\mathrm{t}, 18 \mathrm{H}, \mathrm{CH}_{3}, J_{\mathrm{HH}}=7\right) .{ }^{31} \mathrm{P}\left\{{ }^{1} \mathrm{H}\right\}$ NMR $\left(\mathrm{CD}_{2} \mathrm{Cl}_{2}, 20{ }^{\circ} \mathrm{C}\right) \delta: \mathrm{A}_{2}, 138.5\left(J_{31} \mathrm{p}^{117} \mathrm{Sn}=380.1\right)$. Found: C, 27.22; H, 3.57; N, 2.48; Cl, 19.89. $\mathrm{C}_{24} \mathrm{H}_{38} \mathrm{Cl}_{6} \mathrm{~N}_{2} \mathrm{O}_{6} \mathrm{P}_{2} \mathrm{RuSn}_{2}$ (1063.69) requires $\mathrm{C}, 27.10 ; \mathrm{H}, 3.60 ; \mathrm{N}, 2.63 ; \mathrm{Cl}, 20.00 \%$.)

\section{$\left[\mathrm{Ru}\left(\mathrm{SnH}_{3}\right)(\mathrm{bpy})_{2}\left\{\mathrm{P}(\mathrm{OEt})_{3}\right\}\right] \mathrm{BPh}_{4} 5$}

An excess of $\mathrm{NaBH}_{4}(0.38 \mathrm{~g}, 10 \mathrm{mmol})$ in ethanol $\left(10 \mathrm{~cm}^{3}\right)$ was added to a suspension of $\left[\mathrm{Ru}\left(\mathrm{SnCl}_{3}\right)(\mathrm{bpy})_{2}\left\{\mathrm{P}(\mathrm{OEt})_{3}\right\}\right] \mathrm{BPh}_{4}$ $(1.0 \mathrm{mmol}, 1.12 \mathrm{~g})$ in $50 \mathrm{~cm}^{3}$ of ethanol cooled to $-196{ }^{\circ} \mathrm{C}$. The reaction mixture was left to reach room temperature, stirred for $90 \mathrm{~min}$, and then the solvent removed under reduced pressure. The tin trihydride complex was extracted from the red oil obtained with four $10 \mathrm{~cm}^{3}$ portions of thf. The extracts were evaporated to dryness leaving an oil which was triturated with ethanol $\left(10 \mathrm{~cm}^{3}\right)$. A red solid slowly separated out, which was filtered and crystallised from thf and ethanol; yield $\geq 65 \%$. (IR (KBr) $/ \mathrm{cm}^{-1}: 1726$ (s) 1702 (sh) $v_{\mathrm{SnH}} \cdot{ }^{1} \mathrm{H}$ NMR $\left(\mathrm{thf}_{-\mathrm{d}}^{8}, 20{ }^{\circ} \mathrm{C}\right) \delta: 9.43-6.65(\mathrm{~m}, 36 \mathrm{H}, \mathrm{Ph}+$ bpy), $3.65\left(\mathrm{~m}, 6 \mathrm{H}, \mathrm{CH}_{2}\right), \mathrm{AX}_{3}$ spin system $\left(\mathrm{X}={ }^{1} \mathrm{H}\right), \delta_{\mathrm{X}} 2.38$ $\left(J_{\mathrm{AX}}=0.56, J_{1_{\mathrm{H}^{117} \mathrm{Sn}}}=1080.6\right)\left(3 \mathrm{H}, \mathrm{SnH}_{3}\right), 0.98\left(\mathrm{t}, 9 \mathrm{H}, \mathrm{CH}_{3}, J_{\mathrm{HH}}=\right.$ 7). ${ }^{31} \mathrm{P}\left\{{ }^{1} \mathrm{H}\right\}$ NMR (thf-d $\left.{ }^{8}, 20{ }^{\circ} \mathrm{C}\right) \delta: \mathrm{A}, 140.2\left(J^{31_{\mathrm{p}}{ }^{117} \mathrm{Sn}}=353.3\right)$. $\Lambda_{\mathrm{M}} / \mathrm{S} \mathrm{cm}^{2} \mathrm{~mol}^{-1}$ (acetone) $=113$. Found: C, 58.74; H, 5.45; N, 5.41. $\mathrm{C}_{50} \mathrm{H}_{54} \mathrm{BN}_{4} \mathrm{O}_{3} \mathrm{PRuSn}(1020.55)$ requires $\mathrm{C}, 58.85 ; \mathrm{H}, 5.33 ; \mathrm{N}$, $5.49 \%$.)

\section{$\left[\mathrm{Os}\left(\mathrm{SnH}_{3}\right)(\mathrm{bpy})_{2}\left\{\mathrm{P}(\mathrm{OEt})_{3}\right\}\right] \mathrm{BPh}_{4} 6$}

This complex was prepared exactly like the related ruthenium complex $\mathbf{5}$, but using a reaction time of $2 \mathrm{~h}$. The black solid 
obtained was crystallised from thf and ethanol; yield $\geq 65 \%$. (IR $(\mathrm{KBr}) / \mathrm{cm}^{-1}: 1726(\mathrm{~s}, \mathrm{br}) v_{\mathrm{SnH}} \cdot{ }^{1} \mathrm{H} \mathrm{NMR}\left(\mathrm{thf}_{-} \mathrm{d}^{8}, 20{ }^{\circ} \mathrm{C}\right) \delta: 9.86-$ $6.87\left(\mathrm{~m}, 36 \mathrm{H}, \mathrm{Ph}+\right.$ bpy), 3.92 (qnt, 6H, $\left.\mathrm{CH}_{2}\right), \mathrm{AX}_{3}, \delta_{\mathrm{X}} 2.06$ $\left(J_{\mathrm{AX}}=0.1, J_{1_{\mathrm{H}^{117} \mathrm{Sn}}}=1118.0\right)\left(3 \mathrm{H}, \mathrm{SnH}_{3}\right), 1.21\left(\mathrm{t}, 9 \mathrm{H}, \mathrm{CH}_{3}, J_{\mathrm{HH}}=\right.$ 7). ${ }^{31} \mathrm{P}\left\{{ }^{1} \mathrm{H}\right\}$ NMR (thf-d $\left.8,20{ }^{\circ} \mathrm{C}\right) \delta: \mathrm{A}, 82.8\left(J^{31} \mathrm{p}^{117} \mathrm{Sn}=248.8\right)$. $\Lambda_{\mathrm{M}} / \mathrm{S} \mathrm{cm}^{2} \mathrm{~mol}^{-1}$ (acetone) $=125$. Found: $\mathrm{C}, 54.24 ; \mathrm{H}, 4.76 ; \mathrm{N}$, 4.97. $\mathrm{C}_{50} \mathrm{H}_{54} \mathrm{BN}_{4} \mathrm{O}_{3} \mathrm{OsPSn}(1109.68)$ requires $\mathrm{C}, 54.12 ; \mathrm{H}, 4.90 ; \mathrm{N}$, $5.05 \%$.)

\section{$\mathrm{Ru}\left(\mathrm{SnH}_{3}\right)_{2}(\mathrm{bpy})\left[\mathrm{P}(\mathrm{OEt})_{3}\right]_{2} 7$}

An excess of $\mathrm{NaBH}_{4}(0.19 \mathrm{~g}, 5 \mathrm{mmol})$ in ethanol $\left(5 \mathrm{~cm}^{3}\right)$ was added to a suspension of $\mathrm{Ru}\left(\mathrm{SnCl}_{3}\right)_{2}(\mathrm{bpy})\left[\mathrm{P}(\mathrm{OEt})_{3}\right]_{2} \quad(0.36 \mathrm{~g}$, $0.35 \mathrm{mmol}$ ) in $20 \mathrm{~cm}^{3}$ of ethanol cooled to $-196{ }^{\circ} \mathrm{C}$. The reaction mixture was left to reach room temperature, stirred for $30 \mathrm{~min}$, and then the solvent removed under reduced pressure. The tin trihydride complex was extracted from the residue with four $5 \mathrm{~cm}^{3}$ portions of benzene, and the extracts were evaporated to dryness. Unfortunately, we were not able to transform the red-brown oil obtained into a solid. The spectroscopic data (IR and NMR), however, support the proposed formulation for the complex. (IR $(\mathrm{KBr}) / \mathrm{cm}^{-1}: 1722(\mathrm{~s}, \mathrm{br}) v_{\mathrm{SnH}} \cdot{ }^{1} \mathrm{H} \mathrm{NMR}\left(\mathrm{C}_{6} \mathrm{D}_{6}, 20{ }^{\circ} \mathrm{C}\right) \delta: 9.64-6.68$ (m, 8H, bpy), $4.12\left(\mathrm{~m}, 12 \mathrm{H}, \mathrm{CH}_{2}\right), 3.53\left(\mathrm{~s}, \mathrm{br}, 6 \mathrm{H}, \mathrm{SnH}_{3}, J_{1_{\mathrm{H}}{ }^{119} \mathrm{Sn}}=\right.$ $\left.1083, J_{1_{\mathrm{H}}{ }^{117} \mathrm{Sn}}=1034\right), 1.27\left(\mathrm{t}, 18 \mathrm{H}, \mathrm{CH}_{3}, J_{\mathrm{HH}}=7\right) .{ }^{31} \mathrm{P}\left\{{ }^{1} \mathrm{H}\right\} \mathrm{NMR}$ $\left(\mathrm{C}_{6} \mathrm{D}_{6}, 20^{\circ} \mathrm{C}\right) \delta: \mathrm{A}_{2}, 158.3\left(J_{31} \mathrm{P}^{117} \mathrm{Sn}=203.0\right)$.)

\section{$\left[\mathrm{M}\left(\mathrm{SnMe}_{3}\right)(\mathrm{bpy})_{2}\left\{\mathrm{P}(\mathrm{OEt})_{3}\right\}\right] \mathrm{BPh}_{4}(\mathrm{M}=\mathrm{Ru} \mathbf{8}$, Os 9)}

An excess of $\mathrm{MgBrMe}\left(0.84 \mathrm{mmol}, 280 \mu \mathrm{L}\right.$ of a $3 \mathrm{~mol} \mathrm{dm}^{-3}$ solution in diethylether) was added to a solution of the appropriate $\left[\mathrm{M}\left(\mathrm{SnCl}_{3}\right)(\mathrm{bpy})_{2}\left\{\mathrm{P}(\mathrm{OEt})_{3}\right\}\right] \mathrm{BPh}_{4}$ complex $(0.21 \mathrm{mmol})$ in $20 \mathrm{~cm}^{3}$ of thf cooled to $-196^{\circ} \mathrm{C}$. The reaction mixture was left to reach room temperature and stirred for $20 \mathrm{~min}$. The solvent was removed under reduced pressure to give an oil which was triturated with ethanol $\left(3 \mathrm{~cm}^{3}\right)$ and then an excess of $\mathrm{NaBPh}_{4}(0.36 \mathrm{mmol}$, $123 \mathrm{mg}$ ) in $2 \mathrm{~cm}^{3}$ of ethanol was added. A red (Ru) or black (Os) solid slowly separated out from the resulting solution, which was filtered and crystallised from acetone and ethanol; yield $\geq 70 \%$ for ruthenium and $\geq 65 \%$ for osmium. $\left(8:{ }^{1} \mathrm{H} \mathrm{NMR}\left(\mathrm{CD}_{2} \mathrm{Cl}_{2}, 20{ }^{\circ} \mathrm{C}\right)\right.$ $\delta$ : 9.58-6.73 (m, 36H, Ph + bpy), 3.87, $3.74\left(\mathrm{~m}, 6 \mathrm{H}, \mathrm{CH}_{2}\right), 1.02$ $\left(\mathrm{t}, 9 \mathrm{H}, \mathrm{CH}_{3}\right.$ phos, $\left.J_{\mathrm{HH}}=7\right),-0.50\left(\mathrm{~s}, 9 \mathrm{H}, \mathrm{SnCH}_{3}, J_{1_{\mathrm{H}^{119}} \mathrm{Sn}}=\right.$ 34.2, $\left.J_{1_{\mathrm{H}^{117} \mathrm{Sn}}}=32.7\right) .{ }^{31} \mathrm{P}\left\{{ }^{1} \mathrm{H}\right\} \operatorname{NMR}\left(\mathrm{CD}_{2} \mathrm{Cl}_{2}, 20{ }^{\circ} \mathrm{C}\right) \delta: \mathrm{A}, 140.0$ $\left(J_{31} \mathrm{P}^{117} \mathrm{Sn}=326.9\right) . \Lambda_{\mathrm{M}} / \mathrm{S} \mathrm{cm}{ }^{2} \mathrm{~mol}^{-1}=53.5$. Found: $\mathrm{C}, 59.77 ; \mathrm{H}$, 5.58; N, 5.34. $\mathrm{C}_{53} \mathrm{H}_{60} \mathrm{BN}_{4} \mathrm{O}_{3} \mathrm{PRuSn}$ (1062.63) requires $\mathrm{C}$, 59.91; $\mathrm{H}, 5.69 ; \mathrm{N}, 5.27 \%$. 9: ${ }^{1} \mathrm{H}$ NMR (acetone-d ${ }^{6}, 20{ }^{\circ} \mathrm{C}$ ) $\delta: 9.85-6.73$ $(\mathrm{m}, 36 \mathrm{H}, \mathrm{Ph}+\mathrm{bpy}), 3.81,3.70\left(\mathrm{~m}, 6 \mathrm{H}, \mathrm{CH}_{2}\right), 0.95\left(\mathrm{t}, 9 \mathrm{H}, \mathrm{CH}_{3}\right.$ phos, $\left.J_{\mathrm{HH}}=7\right),-0.54\left(\mathrm{~s}, 9 \mathrm{H}, \mathrm{SnCH}_{3}, J_{1_{\mathrm{H}^{119} \mathrm{Sn}}}=34.3, J_{1_{\mathrm{H}}{ }^{117} \mathrm{Sn}}=\right.$ 32.8). ${ }^{31} \mathrm{P}\left\{{ }^{1} \mathrm{H}\right\}$ NMR (acetone- $\left.\mathrm{d}^{6}, 20{ }^{\circ} \mathrm{C}\right) \delta: \mathrm{A}, 84.7\left(J_{31} \mathrm{p}^{117} \mathrm{Sn}=\right.$ 244.2). $\Lambda_{\mathrm{M}} / \mathrm{S} \mathrm{cm} \mathrm{mol}^{-1}=51.6$. Found: C, 55.19; $\mathrm{H}, 5.20 ; \mathrm{N}$, 4.97. $\mathrm{C}_{53} \mathrm{H}_{60} \mathrm{BN}_{4} \mathrm{O}_{3}$ OsPSn (1151.76) requires $\mathrm{C}, 55.27 ; \mathrm{H}, 5.25 ; \mathrm{N}$, $4.86 \%$.)

\section{$\mathrm{Ru}\left(\mathrm{SnClMe}_{2}\right)_{2}(\mathrm{bpy})\left[\mathrm{P}(\mathrm{OEt})_{3}\right]_{2} 10$}

An excess of $\mathrm{MgBrMe}\left(2.4 \mathrm{mmol}, 800 \mu \mathrm{L}\right.$ of a $3 \mathrm{~mol} \mathrm{dm}^{-3}$ solution in diethylether) was added to a suspension of $\mathrm{Ru}\left(\mathrm{SnCl}_{3}\right)_{2}$ (bpy) $\left[\mathrm{P}(\mathrm{OEt})_{3}\right]_{2}$ complex $(0.30 \mathrm{~g}, 0.29 \mathrm{mmol})$ in $20 \mathrm{~cm}^{3}$ of thf cooled to $-196{ }^{\circ} \mathrm{C}$. The reaction mixture was left to reach room temperature, stirred for $15 \mathrm{~min}$, and then the solvent removed under reduced pressure. The stannyl complex was extracted from the oil obtained with four $5 \mathrm{~cm}^{3}$ portions of benzene. The extracts were evaporated to dryness leaving an oil which was triturated with ethanol $\left(4 \mathrm{~cm}^{3}\right)$. A yellow solid slowly separated out by cooling to the resulting solution $-25{ }^{\circ} \mathrm{C}$, which was filtered and crystallised from toluene and ethanol; yield $\geq 40 \%$. ( ${ }^{1} \mathrm{H}$ NMR (acetone- $\mathrm{d}^{6}$, $\left.20{ }^{\circ} \mathrm{C}\right) \delta: 9.67-7.50(\mathrm{~m}, 8 \mathrm{H}, \mathrm{bpy}), 4.32\left(\mathrm{~m}, 12 \mathrm{H}, \mathrm{CH}_{2}\right), 1.33(\mathrm{t}$, $18 \mathrm{H}, \mathrm{CH}_{3}$ phos, $\left.J_{\mathrm{HH}}=7\right), 0.29\left(\mathrm{~s}, 12 \mathrm{H}, \mathrm{SnCH}_{3}, J_{1_{\mathrm{H}}{ }^{119} \mathrm{Sn}}=34.5\right.$, $J_{1_{\mathrm{H}}{ }^{117} \mathrm{Sn}}=33.0$ ). ${ }^{31} \mathrm{P}\left\{{ }^{1} \mathrm{H}\right\}$ NMR (acetone- $\left.\mathrm{d}^{6}, 20{ }^{\circ} \mathrm{C}\right) \delta: \mathrm{A}_{2}, 144.8$ $\left(J_{31}{ }_{\mathrm{P} 117} \mathrm{Sn}=333.3\right)$. Found: C, 32.76; H, 5.16; N, 3.05; Cl, 7.28. $\mathrm{C}_{26} \mathrm{H}_{50} \mathrm{Cl}_{2} \mathrm{~N}_{2} \mathrm{O}_{6} \mathrm{P}_{2} \mathrm{RuSn}_{2}$ (957.99) requires C, 32.60; H, 5.26; N, $2.92 ; \mathrm{Cl}, 7.40 \%$.)

\section{$\left[\mathbf{R u}\left\{\operatorname{Sn}(\mathrm{C} \equiv \mathrm{CR})_{3}\right\}(\mathrm{bpy})_{2}\left\{\mathrm{P}(\mathrm{OEt})_{3}\right\}\right] \mathrm{BPh}{ }_{4}(\mathrm{R}=p$-tolyl 11, tert-butyl 12)}

An excess of the appropriate lithium acetylide $\mathrm{Li}^{+}[\mathrm{RC} \equiv \mathrm{C}]^{-}$ $\left(1.7 \mathrm{mmol}, 1.15 \mathrm{~cm}^{3}\right.$ of a $1.5 \mathrm{~mol} \mathrm{dm} \mathrm{dm}^{-3}$ solution in thf) was added to a suspension of the $\left[\mathrm{Ru}\left(\mathrm{SnCl}_{3}\right)(\mathrm{bpy})_{2}\left\{\mathrm{P}(\mathrm{OEt})_{3}\right\}\right] \mathrm{BPh}_{4}$ complex (200 mg, $0.18 \mathrm{mmol})$ in $20 \mathrm{~cm}^{3}$ of thf cooled to $-196{ }^{\circ} \mathrm{C}$. The reaction mixture was left to reach room temperature, stirred for $45 \mathrm{~min}$, and then the solvent removed under reduced pressure. The oil obtained was triturated with ethanol $\left(6 \mathrm{~cm}^{3}\right)$ containing an excess of $\mathrm{NaBPh}_{4}(0.36 \mathrm{mmol}, 123 \mathrm{mg})$. By cooling the resulting solution to $-25{ }^{\circ} \mathrm{C}$, a red solid slowly separated out, which was filtered and crystallised from acetone and ethanol; yield $\geq 80 \%$ for 11 and $\geq 65 \%$ for 12. (11: IR $(\mathrm{KBr}) / \mathrm{cm}^{-1}: 2123(\mathrm{~m}) v_{\mathrm{C} \equiv \mathrm{C}}$. ${ }^{1} \mathrm{H}$ NMR $\left(\mathrm{CD}_{2} \mathrm{Cl}_{2}, 20{ }^{\circ} \mathrm{C}\right) \delta: 9.70-6.79(\mathrm{~m}, 48 \mathrm{H}, \mathrm{Ph}+\mathrm{bpy})$, 3.84 (qnt, 6H, $\mathrm{CH}_{2}$ ), 2.34 (s, 9H, $\mathrm{CH}_{3}$ p-tol), 1.04 (t, 9H, $\mathrm{CH}_{3}$ phos, $\left.J_{\mathrm{HH}}=7\right) .{ }^{31} \mathrm{P}\left\{{ }^{1} \mathrm{H}\right\} \operatorname{NMR}\left(\mathrm{CD}_{2} \mathrm{Cl}_{2}, 20{ }^{\circ} \mathrm{C}\right) \delta: \mathrm{A}, 134.0$ $\left(J_{31} \mathrm{p}^{117} \mathrm{Sn}=439.0\right) . \Lambda_{\mathrm{M}} / \mathrm{S} \mathrm{cm}^{2} \mathrm{~mol}^{-1}=51.2$. Found: $\mathrm{C}, 67.67 ; \mathrm{H}$, 5.21; N, 4.23. $\mathrm{C}_{77} \mathrm{H}_{72} \mathrm{BN}_{4} \mathrm{O}_{3} \mathrm{PRuSn}$ (1362.98) requires $\mathrm{C}, 67.85$; $\mathrm{H}, 5.32$; N, 4.11\%. 12: IR (KBr)/ $\mathrm{cm}^{-1}: 2115(\mathrm{~m}) v_{\mathrm{C} \equiv \mathrm{C}}$. ${ }^{1} \mathrm{H}$ NMR (acetone- $\left.\mathrm{d}^{6}, 20{ }^{\circ} \mathrm{C}\right) \delta: 9.76-6.73(\mathrm{~m}, 36 \mathrm{H}, \mathrm{Ph}+\mathrm{bpy}), 3.95$ (qnt, $\left.6 \mathrm{H}, \mathrm{CH}_{2}\right), 1.08\left(\mathrm{t}, 9 \mathrm{H}, \mathrm{CH}_{3}\right.$ phos, $\left.J_{\mathrm{HH}}=7\right), 1.09\left(\mathrm{~s}, 27 \mathrm{H}, \mathrm{CH}_{3}\right.$ $\left.\mathrm{Bu}^{t}\right) .{ }^{31} \mathrm{P}\left\{{ }^{1} \mathrm{H}\right\}$ NMR (acetone-d $\left.\mathrm{d}^{6}, 20{ }^{\circ} \mathrm{C}\right) \delta: \mathrm{A}, 136.7\left(J_{31} \mathrm{p}^{117} \mathrm{Sn}=\right.$ 427.7). $\Lambda_{\mathrm{M}} / \mathrm{S} \mathrm{cm} \mathrm{mol}^{-1}=54.6$. Found: $\mathrm{C}, 64.54 ; \mathrm{H}, 6.15 ; \mathrm{N}$, 4.36. $\mathrm{C}_{68} \mathrm{H}_{78} \mathrm{BN}_{4} \mathrm{O}_{3}$ PRuSn (1260.93) requires $\mathrm{C}, 64.77 ; \mathrm{H}, 6.23 ; \mathrm{N}$, $4.44 \%$.)

\section{$\left[\mathrm{Os}\left\{\mathrm{Sn}(\mathrm{C} \equiv \mathrm{Cp} \text {-tolyl })_{3}\right\}(\text { bpy })_{2}\left\{\mathrm{P}(\mathrm{OEt})_{3}\right\}\right] \mathrm{BPh}_{4} 13$}

The complex was prepared exactly like the related ruthenium complex 11, but using a reaction time of $20 \mathrm{~min}$; yield $\geq 60 \%$. (IR $(\mathrm{KBr}) / \mathrm{cm}^{-1}: 2124(\mathrm{~m}) v_{\mathrm{C} \equiv \mathrm{C}} \cdot{ }^{1} \mathrm{H} \mathrm{NMR}\left(\mathrm{CD}_{2} \mathrm{Cl}_{2}, 20^{\circ} \mathrm{C}\right) \delta: 9.99-6.73$ (m, 48H, Ph + bpy), 3.91 (qnt, 6H, $\mathrm{CH}_{2}$ ), 2.30 (s, 9H, $\mathrm{CH}_{3} p$-tol), 1.04 (t, 9H, $\mathrm{CH}_{3}$ phos, $\left.J_{\mathrm{HH}}=7\right) .{ }^{31} \mathrm{P}\left\{{ }^{1} \mathrm{H}\right\} \operatorname{NMR}\left(\mathrm{CD}_{2} \mathrm{Cl}_{2}, 20{ }^{\circ} \mathrm{C}\right)$ $\delta$ : A, $79.2\left(J_{31} \mathrm{p}^{117} \mathrm{Sn}=304.0\right) . \Lambda_{\mathrm{M}} / \mathrm{S} \mathrm{cm}^{2} \mathrm{~mol}^{-1}=53.8$. Found: $\mathrm{C}$, 63.52; $\mathrm{H}, 4.93 ; \mathrm{N}, 3.98 . \mathrm{C}_{77} \mathrm{H}_{72} \mathrm{BN}_{4} \mathrm{O}_{3} \mathrm{OsPSn}$ (1452.11) requires $\mathrm{C}$, $63.69 ; \mathrm{H}, 5.00 ; \mathrm{N}, 3.86 \%$.)

\section{$\mathrm{Ru}\left[\mathrm{Sn}(\mathrm{C} \equiv \mathrm{CR})_{3}\right]_{2}(\mathrm{~N}-\mathrm{N})\left[\mathrm{P}(\mathrm{OEt})_{3}\right]_{2}$ 14-17 $(\mathrm{N}-\mathrm{N}=$ bpy 14,15 , phen 16, 17; $\mathrm{R}=$ p-tolyl 14, 16, $\mathrm{Bu}^{t} 15,17$ )}

An excess of lithium acetylide $\mathrm{Li}^{+}[\mathrm{RC} \equiv \mathrm{C}]^{-}\left(1.7 \mathrm{mmol}, 1.15 \mathrm{~cm}^{3}\right.$ of a $1.5 \mathrm{~mol} \mathrm{dm}{ }^{-3}$ solution in thf) was added to a suspension of the appropriate $\mathrm{Ru}\left(\mathrm{SnCl}_{3}\right)_{2}(\mathrm{~N}-\mathrm{N})\left[\mathrm{P}(\mathrm{OEt})_{3}\right]_{2}$ complex $(0.17 \mathrm{mmol})$ in $20 \mathrm{~cm}^{3}$ of thf cooled to $-196{ }^{\circ} \mathrm{C}$. The reaction mixture was left to reach room temperature, stirred for $30 \mathrm{~min}$, and then the solvent removed under reduced pressure. The stannyl complexes 
were extracted from the oil obtained with four $5 \mathrm{~cm}^{3}$ portions of benzene. The extracts were evaporated to dryness leaving an oil which was treated with ethanol $\left(5 \mathrm{~cm}^{3}\right)$. A yellow solid slowly separated out from the resulting solution, which was cooled to $-25{ }^{\circ} \mathrm{C}$ to complete the precipitation. The solid was filtered and crystallised from ethanol; yield $\geq 70 \%$ for 14,16 and $\geq 60 \%$ for 15, 17. (14: IR (KBr) $/ \mathrm{cm}^{-1}: 2126$ (s) $v_{\mathrm{C} \equiv \mathrm{C}} \cdot{ }^{1} \mathrm{H}$ NMR (acetone- $\mathrm{d}^{6}$, $\left.20{ }^{\circ} \mathrm{C}\right) \delta: 9.45-6.91(\mathrm{~m}, 32 \mathrm{H}, \mathrm{Ph}+\mathrm{bpy}), 4.30\left(\mathrm{~m}, 12 \mathrm{H}, \mathrm{CH}_{2}\right), 2.30$ (s, $18 \mathrm{H}, \mathrm{CH}_{3} p$-tol), $1.40\left(\mathrm{t}, 18 \mathrm{H}, \mathrm{CH}_{3}\right.$ phos, $\left.J_{\mathrm{HH}}=7\right) .{ }^{31} \mathrm{P}\left\{{ }^{1} \mathrm{H}\right\}$ NMR (acetone-d $\left.{ }^{6}, 20{ }^{\circ} \mathrm{C}\right) \delta: \mathrm{A}_{2}, 149.2\left(J^{31_{1} \mathrm{p}^{117} \mathrm{Sn}}=286.3\right)$. Found: $\mathrm{C}, 60.01 ; \mathrm{H}, 5.43 ; \mathrm{N}, 1.76 . \mathrm{C}_{76} \mathrm{H}_{80} \mathrm{~N}_{2} \mathrm{O}_{6} \mathrm{P}_{2} \mathrm{RuSn}_{2}$ (1517.88) requires C, 60.14; H, 5.31; N, 1.85\%. 15: IR (KBr)/ $\mathrm{cm}^{-1}: 2120(\mathrm{~m}) v_{\mathrm{C} \equiv \mathrm{C}}{ }^{1} \mathrm{H}$ NMR (acetone- $\left.\mathrm{d}^{6}, 20^{\circ} \mathrm{C}\right) \delta: 9.60-7.45(\mathrm{~m}, 8 \mathrm{H}$, bpy), $4.22(\mathrm{~m}, 12 \mathrm{H}$, $\left.\mathrm{CH}_{2}\right), 1.12\left(\mathrm{t}, 18 \mathrm{H}, \mathrm{CH}_{3}\right.$ phos, $\left.J_{\mathrm{HH}}=7\right), 1.04\left(\mathrm{~s}, 54 \mathrm{H}, \mathrm{CH}_{3} \mathrm{Bu}^{t}\right)$.

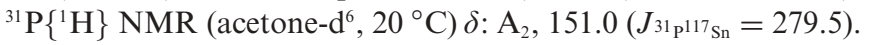
Found: C, 53.25; H, 6.98; N, 2.03. $\mathrm{C}_{58} \mathrm{H}_{92} \mathrm{~N}_{2} \mathrm{O}_{6} \mathrm{P}_{2} \mathrm{RuSn}_{2}$ (1313.77) requires $\mathrm{C}, 53.03 ; \mathrm{H}, 7.06 ; \mathrm{N}, 2.13 \%$. 16: $\mathrm{IR}(\mathrm{KBr}) / \mathrm{cm}^{-1}: 2120$ (m) $v_{\mathrm{C} \equiv \mathrm{C}} \cdot{ }^{1} \mathrm{H}$ NMR (acetone-d $\left.\mathrm{d}^{6}, 20{ }^{\circ} \mathrm{C}\right) \delta$ : 10.48-6.90 (m, 32H, $\mathrm{Ph}+$ phen), 4.40, $3.70\left(\mathrm{~m}, 12 \mathrm{H}, \mathrm{CH}_{2}\right), 2.29,2.28,2.24(\mathrm{~s}, 18 \mathrm{H}$, $\mathrm{CH}_{3}$ p-tol), 1.46, 1.44, 0.77 (t, $18 \mathrm{H}, \mathrm{CH}_{3}$ phos, $\left.J_{\mathrm{HH}}=7\right) .{ }^{31} \mathrm{P}\left\{{ }^{1} \mathrm{H}\right\}$

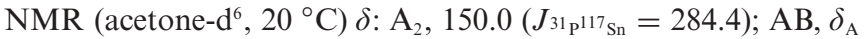
$146.5, \delta_{\mathrm{B}} 135.0, J_{\mathrm{AB}}=50.0, J_{\mathrm{PA}^{117} \mathrm{Sn}}=339.3, J_{\mathrm{PB}^{117} \mathrm{Sn}}=2447.7$, $J_{\mathrm{PA}^{117} \mathrm{Sn}}=469.2, J_{\mathrm{PB}^{117} \mathrm{Sn}}=294.4$. Found: $\mathrm{C}, 60.54 ; \mathrm{H}, 5.36 ; \mathrm{N}$, 1.75. $\mathrm{C}_{78} \mathrm{H}_{80} \mathrm{~N}_{2} \mathrm{O}_{6} \mathrm{P}_{2} \mathrm{RuSn}_{2}$ (1541.90) requires $\mathrm{C}, 60.76 ; \mathrm{H}, 5.23 ; \mathrm{N}$, 1.82\%. 17: IR (KBr) $/ \mathrm{cm}^{-1}: 2120(\mathrm{~m}) v_{\mathrm{C} \equiv \mathrm{C}} \cdot{ }^{1} \mathrm{H}$ NMR (acetone- $\mathrm{d}^{6}$, $\left.20{ }^{\circ} \mathrm{C}\right) \delta: 9.74-7.97(\mathrm{~m}, 8 \mathrm{H}$, phen $), 4.30\left(\mathrm{~m}, 12 \mathrm{H}, \mathrm{CH}_{2}\right), 1.43(\mathrm{t}$, $18 \mathrm{H}, \mathrm{CH}_{3}$ phos, $\left.J_{\mathrm{HH}}=7\right), 0.99\left(\mathrm{~s}, 54 \mathrm{H}, \mathrm{CH}_{3} \mathrm{Bu}^{t}\right) .{ }^{31} \mathrm{P}\left\{{ }^{1} \mathrm{H}\right\}$ NMR (acetone- $\left.\mathrm{d}^{6}, 20^{\circ} \mathrm{C}\right) \delta: \mathrm{A}_{2}, 151.5\left(J_{31} \mathrm{p}^{117} \mathrm{Sn}=278.8\right)$. Found: C, 53.64; $\mathrm{H}, 7.04 ; \mathrm{N}, 2.14 . \mathrm{C}_{60} \mathrm{H}_{92} \mathrm{~N}_{2} \mathrm{O}_{6} \mathrm{P}_{2} \mathrm{RuSn}_{2}$ (1337.79) requires $\mathrm{C}, 53.87$; $\mathrm{H}, 6.93 ; \mathrm{N}, 2.09 \%$.)

\section{Crystallographic analysis of $\left[\mathrm{Ru}\left(\mathrm{SnMe}_{3}\right)(\mathrm{bpy})_{2}\left\{\mathrm{P}(\mathrm{OEt})_{3}\right\}\right] \mathrm{BPh}_{4} 8 \dagger$}

Data were collected by a SIEMENS Smart CCD area-detector diffractometer with graphite-monochromated Mo-K $\alpha$ radiation. Absorption correction was carried out with SADABS. ${ }^{11}$ The structure was solved with the Oscail program ${ }^{12}$ by direct methods and refined by full-matrix least-squares based on $F^{2} \cdot{ }^{13}$ Non-hydrogen atoms were refined with anisotropic displacement parameters. Hydrogen atoms were included in idealised positions and refined with isotropic displacement parameters. Atomic scattering factors and anomalous dispersion corrections for all atoms were taken from the International Tables for X-ray Crystallography. ${ }^{14}$ Details of crystal data and structural refinement are given in Table 1.

\section{Results and discussion}

\section{Synthesis of stannyl complexes}

Cationic monophosphine complexes $\left[\mathrm{MCl}(\mathrm{bpy})_{2} \mathrm{P}\right]^{+}$react with $\mathrm{SnCl}_{2}$ to give trichlorostannyl $\left[\mathrm{M}\left(\mathrm{SnCl}_{3}\right)(\mathrm{bpy})_{2} \mathrm{P}\right]^{+} \mathbf{1}, \mathbf{2}$ derivatives, which were isolated as $\mathrm{BPh}_{4}$ salts and characterised (Scheme 1).

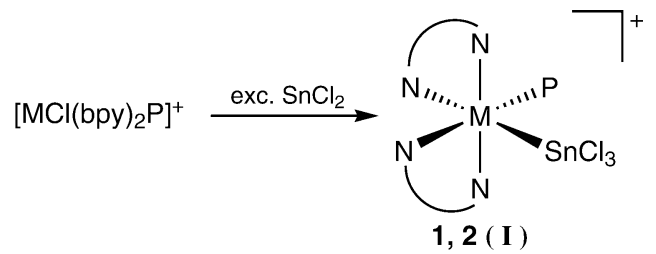

Scheme $1 \quad \mathrm{M}=\mathrm{Ru}, \mathrm{P}=\mathrm{P}(\mathrm{OEt})_{3} \mathbf{1 a}, \mathrm{PPh}(\mathrm{OEt})_{2} \mathbf{1 b} ; \mathrm{M}=\mathrm{Os}, \mathrm{P}=\mathrm{P}(\mathrm{OEt})_{3} 2$.

Table 1 Crystal data and structure refinement for $\mathbf{8} \cdot \mathrm{Me}_{2} \mathrm{CO}$

\begin{tabular}{|c|c|}
\hline Identification code & 8. $\mathrm{Me}_{2} \mathrm{CO}$ \\
\hline Empirical formula & $\mathrm{C}_{56} \mathrm{H}_{66} \mathrm{BN}_{4} \mathrm{O}_{4} \mathrm{PRuSn}$ \\
\hline Formula weight & 1120.67 \\
\hline Temperature/K & $293(2)$ \\
\hline Wavelength/Å & 0.71073 \\
\hline Crystal system, space group & Triclinic, $P \overline{1}$ \\
\hline \multicolumn{2}{|l|}{ Unit cell dimensions: } \\
\hline$a / \AA ̊$ & $12.2313(10)$ \\
\hline$b / \AA$ & $13.9015(11)$ \\
\hline$c / \AA$ & $16.1270(13)$ \\
\hline$a /{ }^{\circ}$ & $93.132(2)$ \\
\hline$\beta /{ }^{\circ}$ & $95.390(2)$ \\
\hline$\gamma /{ }^{\circ}$ & $94.700(2)$ \\
\hline Volume $/ \AA^{3}$ & $2715.4(4)$ \\
\hline$Z$ & 2 \\
\hline Density (calculated) $/ \mathrm{Mg} \mathrm{m}^{-3}$ & 1.371 \\
\hline Absorption coefficient $/ \mathrm{mm}^{-1}$ & 0.815 \\
\hline$F(000)$ & 1152 \\
\hline Crystal size/mm & $0.35 \times 0.25 \times 0.15$ \\
\hline$\theta$ range for data collection $/^{\circ}$ & 1.47 to 28.04 \\
\hline Index ranges & $-15 \leq h \leq 16 ;-13 \leq k \leq 18 ;-21 \leq l \leq 21$ \\
\hline Reflections collected & $1804 \overline{9}$ \\
\hline Independent reflections & $12534[R(\mathrm{int})=0.0762]$ \\
\hline Reflections observed $(>2 \sigma)$ & 5206 \\
\hline Data completeness & 0.951 \\
\hline Absorption correction & Semi-empirical from equivalents \\
\hline Max. and min. transmission & 1.000 and 0.539 \\
\hline Data/restraints/parameters & $12534 / 0 / 621$ \\
\hline Goodness-of-fit on $F^{2}$ & 0.820 \\
\hline Final $\mathrm{R}$ indices $[\mathrm{I}>2 \sigma(I)]$ & $R_{1}=0.0575 w R_{2}=0.1100$ \\
\hline$R$ indices (all data) & $R_{1}=0.1537 w R_{2}=0.1439$ \\
\hline Largest diff. peak and hole/e $\AA^{-3}$ & 0.531 and -0.868 \\
\hline
\end{tabular}


The related cationic tris(phosphine) $\left[\mathrm{RuCl}(\mathrm{N}-\mathrm{N}) \mathrm{P}_{3}\right]^{+}$complexes react with $\mathrm{SnCl}_{2} \cdot 2 \mathrm{H}_{2} \mathrm{O}$ to give the bis(trichlorostannyl) $\mathrm{Ru}\left(\mathrm{SnCl}_{3}\right)_{2}(\mathrm{~N}-\mathrm{N}) \mathrm{P}_{2} \mathbf{3}, 4$ derivatives in about $60 \%$ yield (Scheme 2 ).

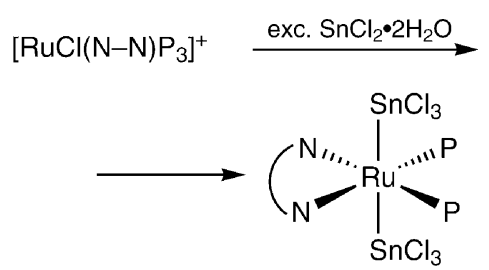

3a, 4 ( II )

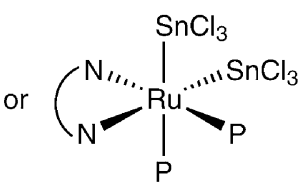

3b ( III )
Scheme $2 \mathrm{~N}-\mathrm{N}=$ bpy, $\mathrm{P}=\mathrm{P}(\mathrm{OEt})_{3}$ 3a, $\mathrm{PPh}(\mathrm{OEt})_{2} \mathbf{3 b} ; \mathrm{N}-\mathrm{N}=$ phen, $\mathrm{P}=$ $\mathrm{P}(\mathrm{OEt})_{3} 4$.

The formation of bis(trichlorostannyl) complexes $\mathbf{3}, \mathbf{4}$, starting from monochloro $\left[\mathrm{RuCl}(\mathrm{N}-\mathrm{N}) \mathrm{P}_{3}\right]^{+}$precursors is rather unexpected, and probably involves several intermediates. In order to obtain information on the reaction path, we monitored the progress of the reaction by NMR spectroscopy, but unfortunately no intermediate was observed and therefore no reasonable path may be proposed for the formation of bis(stannyl) derivatives $\mathbf{3}, \mathbf{4}$.

Treatment of monostannyl $\left[\mathrm{M}\left(\mathrm{SnCl}_{3}\right)(\mathrm{N}-\mathrm{N})_{2} \mathrm{P}\right]^{+} \mathbf{1 a}, \mathbf{2}$ cations with $\mathrm{NaBH}_{4}$ in ethanol gave the trihydride $\left[\mathrm{M}\left(\mathrm{SnH}_{3}\right)(\mathrm{N}-\mathrm{N})_{2} \mathrm{P}\right]^{+}$ $\mathbf{5}, \mathbf{6}$ derivatives, which were isolated as $\mathrm{BPh}_{4}$ salts in good yields (Scheme 3).

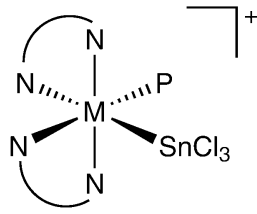

1a, 2<smiles>FP1([SiH3])([SiH3])NCCN1[AsH3-]</smiles>

$3 a$

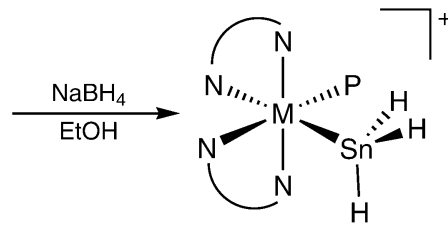

5, 6 ( IV)



Scheme $3 \mathrm{M}=\mathrm{Ru}$ 5, Os 6; $\mathrm{N}-\mathrm{N}=$ bpy; $\mathrm{P}=\mathrm{P}(\mathrm{OEt})_{3}$.

Crucial for successful synthesis was to start the reaction at a low temperature $\left(-196^{\circ} \mathrm{C}\right)$ and to extract the trihydridestannyl compounds with thf. Otherwise, large amounts of decomposition products were obtained, which prevented purification of the complexes.

The bis(trichlorostannyl) $\mathrm{Ru}\left(\mathrm{SnCl}_{3}\right)_{2}(\mathrm{~N}-\mathrm{N}) \mathrm{P}_{2} 3$ complex also reacted with $\mathrm{NaBH}_{4}$ (Scheme 3) to afford an oily product which could not be separated as a solid. However, the spectroscopic data strongly support its formulation as a bis(trihydridestannyl) $\mathrm{Ru}\left(\mathrm{SnH}_{3}\right)_{2}(\mathrm{~N}-\mathrm{N}) \mathrm{P}_{2} 7$ derivative.

The reaction with $\mathrm{NaBH}_{4}$ proceeded, in both cases, with the substitution of all the chlorides by $\mathrm{H}^{-}$in the $\mathrm{SnCl}_{3}$ group, giving the trihydride $\mathrm{SnH}_{3}$ ligand. These results also highlight the fact that polypyridines, in mixed-ligand complexes with phosphites, can stabilise tin trihydride ligands. In addition, bis(stannyl) complexes 3, 4, 7 can also be prepared with polypyridine as the supporting ligand, including the first example of a metal complex containing two trihydridestannyl groups. Although transition metal bis(stannyl) compounds are known, ${ }^{5,15}$ few involve ruthenium as a central metal, and none contain two $\mathrm{SnH}_{3}$ ligands.

Substitution of all the chlorides in trichlorostannyl [M] $-\mathrm{SnCl}_{3}$ complexes 1a, 2 also proceeded in the presence of $\mathrm{MgBrMe}$, giving the trimethylstannyl $\left[\mathrm{M}\left(\mathrm{SnMe}_{3}\right)(\mathrm{N}-\mathrm{N})_{2} \mathrm{P}\right]^{+} \mathbf{8}, 9$ derivatives, which were separated as $\mathrm{BPh}_{4}$ salts and characterised (Scheme 4). However, in the case of the bis(trichlorostannyl) $\mathrm{Ru}\left(\mathrm{SnCl}_{3}\right)_{2}(\mathrm{~N}-\mathrm{N}) \mathrm{P}_{2}$ 3a precursor, the reaction with the Grignard reagent $\mathrm{MgBrMe}$ proceeded with the substitution of only two $\mathrm{Cl}^{-}$, yielding the bis(dimethylstannyl) $\mathrm{Ru}\left(\mathrm{SnClMe}_{2}\right)_{2}(\mathrm{~N}-\mathrm{N}) \mathrm{P}_{2} \mathbf{1 0}$ complex (Scheme 4). The use of an excess of MgBrMe or of a long reaction time did not lead to the substitution of all three chlorides in $\mathrm{SnCl}_{3}$, and the di-substituted $\mathrm{Ru}\left(\mathrm{SnClMe}_{2}\right)_{2}(\mathrm{~N}-\mathrm{N}) \mathrm{P}_{2}$ $\mathbf{1 0}$ derivative was the only isolated product.
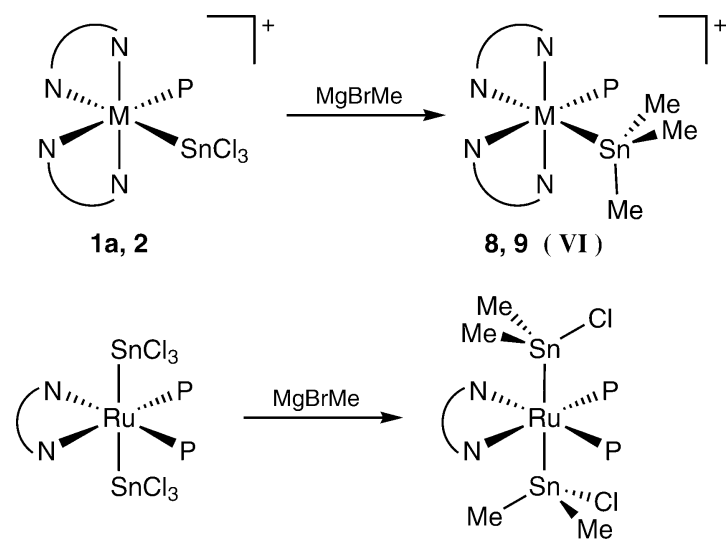

$3 a$

10 ( VII )

Scheme $4 \mathrm{M}=\mathrm{Ru}$ 8, Os 9; $\mathrm{N}-\mathrm{N}=$ bpy; $\mathrm{P}=\mathrm{P}(\mathrm{OEt})_{3}$.

Complexes containing the trimethylstannyl ligand $\mathrm{SnMe}_{3}$ are known for ruthenium and osmium central metals, ${ }^{2 f, 2 h}$ and are often obtained by oxidative addition of $\mathrm{Me}_{3} \mathrm{SnH}$ or $\mathrm{Me}_{3} \mathrm{SnCl}$ species on appropriate complex precursors. Nucleophilic substitution of chloride in the $\mathrm{SnCl}_{3}$ ligand is thus an interesting protocol for the synthesis of tin-organostannyl derivatives.

Treatment of both mono(trichlorostannyl) $\left[\mathrm{M}\left(\mathrm{SnCl}_{3}\right)(\mathrm{N}-\right.$ $\left.\mathrm{N})_{2} \mathrm{P}\right]^{+}$and bis(trichlorostannyl) $\mathrm{M}\left(\mathrm{SnCl}_{3}\right)_{2}(\mathrm{~N}-\mathrm{N}) \mathrm{P}_{2}$ complexes with lithium acetylide afforded tris(alkynyl)stannyl [M $\{\mathrm{Sn}-$ $\left.\left.(\mathrm{C} \equiv \mathrm{CR})_{3}\right\}(\mathrm{N}-\mathrm{N})_{2} \mathrm{P}\right]^{+} \quad \mathbf{1 1 - 1 3}$ and bis[tris(alkynyl)stannyl] $\mathrm{Ru}\left\{\mathrm{Sn}(\mathrm{C} \equiv \mathrm{CR})_{3}\right\}_{2}(\mathrm{~N}-\mathrm{N}) \mathrm{P}_{2}$ 14-17 derivatives, respectively, which were isolated in the solid state and characterised (Scheme 5). The reaction proceeded with the substitution of all the chlorides in $\mathrm{SnCl}_{3}$, yielding the unprecedented complexes containing tris(alkynyl)stannyl as ligand..$^{1-3}$ In fact, although numerous $\mathrm{SnR}_{3}$ stannyl ligands with various substituents are known, ${ }^{1-3}$ the only one containing three alkynyl groups has been obtained in our laboratory. ${ }^{16}$

\section{Characterisation of $[\mathrm{M}]-\mathrm{SnCl}_{3}$ and $[\mathrm{M}]-\mathrm{SnH}_{3}$ species}

Trichlorostannyl $\left[\mathrm{M}\left(\mathrm{SnCl}_{3}\right)(\mathrm{N}-\mathrm{N})_{2} \mathrm{P}_{\mathrm{BPh}}\right.$ 1, 2 complexes were isolated as orange $(\mathrm{Ru})$ or brown $(\mathrm{Os})$ solids, stable in air and in solutions of polar organic solvents, where they behave as $1: 1$ 


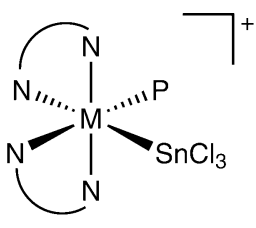

1a, 2
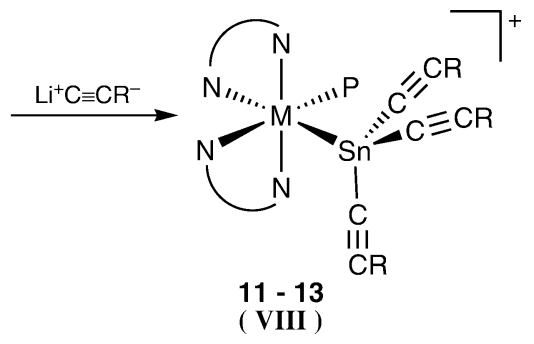

$\mathrm{M}=\mathrm{Ru} 11,12$, Os 13; N-N = bpy; $\mathrm{R}=p$-tolyl 11, 13, $\mathrm{Bu}^{t} 12$
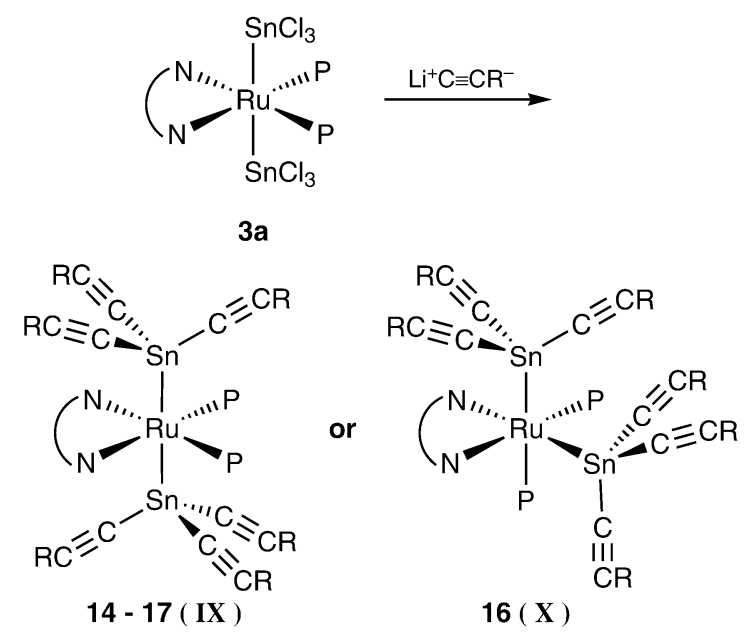

$\mathrm{N}-\mathrm{N}=$ bpy 14,15 , phen 16,$17 ; \mathrm{R}=p$-tolyl $14,16, \mathrm{Bu}^{t} 15,17$

Scheme $5 \quad \mathrm{P}=\mathrm{P}(\mathrm{OEt})_{3}$.

electrolytes. ${ }^{17}$ The related bis(trichlorostannyl) $\mathrm{Ru}\left(\mathrm{SnCl}_{3}\right)_{2}(\mathrm{~N}-$ N) $P_{2}$ 3, 4 complexes are red-brown stable solids and soluble in common organic solvents, where they behave as non-electrolytes. The analytical and spectroscopic data (IR and ${ }^{1} \mathrm{H},{ }^{31} \mathrm{P},{ }^{119} \mathrm{Sn}$ NMR; Experimental and Table 2) support the proposed formulations and allow a geometry in solution to be established.

The IR spectra of [M] $-\mathrm{SnCl}_{3}$ complexes 1-4 show the bands characteristic of polypyridine (1600-1300 $\left.\mathrm{cm}^{-1}\right)$ and phosphite ligands $\left(v_{\mathrm{PO}}\right.$ at $\left.1200-1050 \mathrm{~cm}^{-1}\right)$, the presence of which was confirmed by the ${ }^{1} \mathrm{H}$ NMR spectra of the compounds.

The ${ }^{31} \mathrm{P}$ spectra of monostannyl $\left[\mathrm{M}\left(\mathrm{SnCl}_{3}\right)(\mathrm{N}-\mathrm{N})_{2} \mathrm{P} \mathrm{BPh}_{4} \mathbf{1}, \mathbf{2}\right.$ derivatives were sharp singlets with characteristic satellites, due to coupling with the ${ }^{119} \mathrm{Sn}$ and ${ }^{117} \mathrm{Sn}$ nuclei of the $\mathrm{SnCl}_{3}$ ligand. Instead, the ${ }^{119} \mathrm{Sn}$ NMR spectra appeared as doublets between -126.1 and $-464.1 \mathrm{ppm}$, due to coupling with one phosphorus nucleus of the phosphite, fitting the proposed formulation for the complexes. In addition, by comparison with literature data, ${ }^{18}$ the values for the $J_{119_{\mathrm{nn}}{ }^{31} \mathrm{p}}$ at $344-546 \mathrm{~Hz}$ in our complexes also suggested the mutually cis position of the phosphite and stannyl ligands. On the basis of these data, cis geometry (I, Scheme 1) is proposed for monostannyl derivatives $\mathbf{1}$ and $\mathbf{2}$.

The ${ }^{31} \mathrm{P}$ and ${ }^{119} \mathrm{Sn}$ NMR spectra of the bis(trichlorostannyl) $\mathrm{Ru}\left(\mathrm{SnCl}_{3}\right)_{2}(\mathrm{~N}-\mathrm{N}) \mathrm{P}_{2} \mathbf{3}, \mathbf{4}$ derivatives depend on the nature of the phosphite ligands. In the temperature range between +20 and $-80{ }^{\circ} \mathrm{C}$, the ${ }^{31} \mathrm{P}$ NMR spectra of $\mathrm{P}(\mathrm{OEt})_{3}$ complexes $\mathbf{3 a}$ and $\mathbf{4}$ showed a sharp singlet with the ${ }^{119} \mathrm{Sn}$ and ${ }^{117} \mathrm{Sn}$ satellites, suggesting the magnetic equivalence of the two phosphite ligands. Conversely, the ${ }^{119} \mathrm{Sn}$ NMR spectra appeared as sharp triplets, due to coupling with the two equivalent phosphorus nuclei of the phosphites, indicating the magnetic equivalence of the two $\mathrm{SnCl}_{3}$ groups. In the ${ }^{119} \mathrm{Sn}$ spectrum of complex $\mathrm{Ru}\left(\mathrm{SnCl}_{3}\right)_{2}(\mathrm{bpy})\left[\mathrm{P}(\mathrm{OEt})_{3}\right]_{2}$ 3a the satellites also appeared, due to coupling with the ${ }^{117} \mathrm{Sn}$ nucleus, with a $J_{119} \mathrm{Sn}^{117} \mathrm{sn}$ value of $24990 \mathrm{~Hz}$. The very high value ${ }^{19}$ of this coupling constant indicates the mutually trans position of the two stannyl groups. On the basis of these data, cis-trans geometry of type II (Scheme 2) is proposed for bis(trichlorostannyl) complexes 3a and 4. In addition, the observed values of $390.5-389.5 \mathrm{~Hz}$ for the $J_{119_{\mathrm{Sn}^{3}} 1_{\mathrm{P}}}$ of $\mathbf{3 a}$ and $\mathbf{4}$ confirm the mutually cis position of the phosphite and $\mathrm{SnCl}_{3}$ ligands, as in geometry II.

In the temperature range between +20 and $-80{ }^{\circ} \mathrm{C}$, the ${ }^{31} \mathrm{P}$ $\mathrm{NMR}$ spectrum of $\mathrm{Ru}\left(\mathrm{SnCl}_{3}\right)_{2}(\mathrm{bpy})\left[\mathrm{PPh}(\mathrm{OEt})_{2}\right]_{2}$ 3b showed an $\mathrm{AB}$ quartet at 166-154 ppm, with the characteristic satellites of the ${ }^{119} \mathrm{Sn}$ and ${ }^{117} \mathrm{Sn}$ nuclei of the $\mathrm{SnCl}_{3}$ groups, suggesting the magnetic non-equivalence of the two phosphite ligands. The ${ }^{119} \mathrm{Sn}$ NMR spectra appeared as a complicated pattern, which can be simulated using two $\mathrm{ABM}$ spin systems $\left(\mathrm{M}={ }^{119} \mathrm{Sn}\right)$ with the parameters reported in Table 2. On the basis of these data, a cis-cis geometry of type III would be present in the bis(stannyl) derivative $\mathbf{3 b}$.

Trihydridestannyl $\left[\mathrm{M}\left(\mathrm{SnH}_{3}\right)(\mathrm{bpy})_{2}\left\{\mathrm{P}(\mathrm{OEt})_{3}\right\}\right] \mathrm{BPh}_{4}$ complexes are red (5) or black (6) solids, stable in air and moderately stable in solutions of acetone and thf, where they behave as 1 : 1 electrolytes. ${ }^{17}$ Besides the absorptions of the bpy and $\mathrm{P}(\mathrm{OEt})_{3}$ ligands and the $\mathrm{BPh}_{4}{ }^{-}$anion, the IR spectra showed two bands at $1726-1702 \mathrm{~cm}^{-1}$, attributed to the $v_{\mathrm{SnH}}$ of the trihydridestannyl ligand. Diagnostic for the presence of the $\mathrm{SnH}_{3}$ group, however, were both the ${ }^{1} \mathrm{H}$ and ${ }^{119} \mathrm{Sn}$ NMR spectra of the complexes. A multiplet at $2.38(5)$ or $2.06(6) \mathrm{ppm}$, with the characteristic satellites due to coupling with the ${ }^{119} \mathrm{Sn}$ and ${ }^{117} \mathrm{Sn}$ nuclei, was present in the ${ }^{1} \mathrm{H}$ NMR spectra of $\mathbf{5}$ and $\mathbf{6}$, and was attributed to the resonance of the $\mathrm{SnH}_{3}$ group. As the ${ }^{31} \mathrm{P}$ spectra were singlets, due to the presence of only one phosphite ligand, the hydride multiplet was simulated with an $\mathrm{AX}_{3}$ model $\left(\mathrm{A}={ }^{31} \mathrm{P}, \mathrm{X}={ }^{1} \mathrm{H}\right)$ according to the parameters listed in Experimental, fitting the presence of the $\mathrm{SnH}_{3}$ ligand. Also the proton-coupled ${ }^{119} \mathrm{Sn}$ NMR spectra of $\mathbf{5}$ and $\mathbf{6}$ appeared as a doublet of quartets, simulable with an $\mathrm{AMX}_{3}$ model and fitting the proposed formulation. Lastly, the values of $J_{119_{\mathrm{Sn}^{\mathrm{B}}} 1_{\mathrm{P}}}$ of $370.3 \mathrm{~Hz}$ for ruthenium complex 5 and of $259.8 \mathrm{~Hz}$ for osmium 6 suggest the mutually cis position of the phosphite and $\mathrm{SnH}_{3}$ groups, as in type IV geometry (Scheme 3).

Bis(trihydridestannyl) $\mathrm{Ru}\left(\mathrm{SnH}_{3}\right)_{2}(\mathrm{bpy})\left[\mathrm{P}(\mathrm{OEt})_{3}\right]_{2}$ complex 7 is a reddish-brown oil, the IR spectrum of which showed a broad strong band at $1722 \mathrm{~cm}^{-1}$, attributed to the $v_{\mathrm{SnH}}$ of the $\mathrm{SnH}_{3}$ groups. The ${ }^{1} \mathrm{H}$ NMR spectrum confirmed the presence of the tin trihydride ligand, showing a slightly broad singlet at $3.53 \mathrm{ppm}$, with the characteristic satellites of the ${ }^{119} \mathrm{Sn}$ and ${ }^{117} \mathrm{Sn}$ nuclei. The proton-coupled ${ }^{119} \mathrm{Sn}$ NMR spectrum also appeared as a triplet of quartets, fitting the presence of the $\mathrm{SnH}_{3}$ group. The multiplicity of the signals also suggested the magnetic equivalence of the two $\mathrm{SnH}_{3}$ groups. However, in the spectrum the satellites due to ${ }^{119} \mathrm{Sn}-$ ${ }^{117} \mathrm{Sn}$ coupling were not observed, so that the mutually cis or trans position of the two $\mathrm{SnH}_{3}$ groups could not be ascertained. In the temperature range between +20 and $-80^{\circ} \mathrm{C}$, the ${ }^{31} \mathrm{P}$ NMR spectra were sharp singlets with the characteristic ${ }^{119} \mathrm{Sn}$ and ${ }^{117} \mathrm{Sn}$ satellites, suggesting the magnetic equivalence of the two phosphite ligands, which would be $\left(J_{31 \mathrm{p} 119} 9_{\mathrm{Sn}}=217 \mathrm{~Hz}\right)$ in a mutually cis position with respect to the two $\mathrm{SnH}_{3}$ ligands. These data do not allow us to 
Table $2{ }^{13} \mathrm{C}\left\{{ }^{1} \mathrm{H}\right\}$ and ${ }^{119} \mathrm{Sn}$ NMR data for ruthenium and osmium complexes

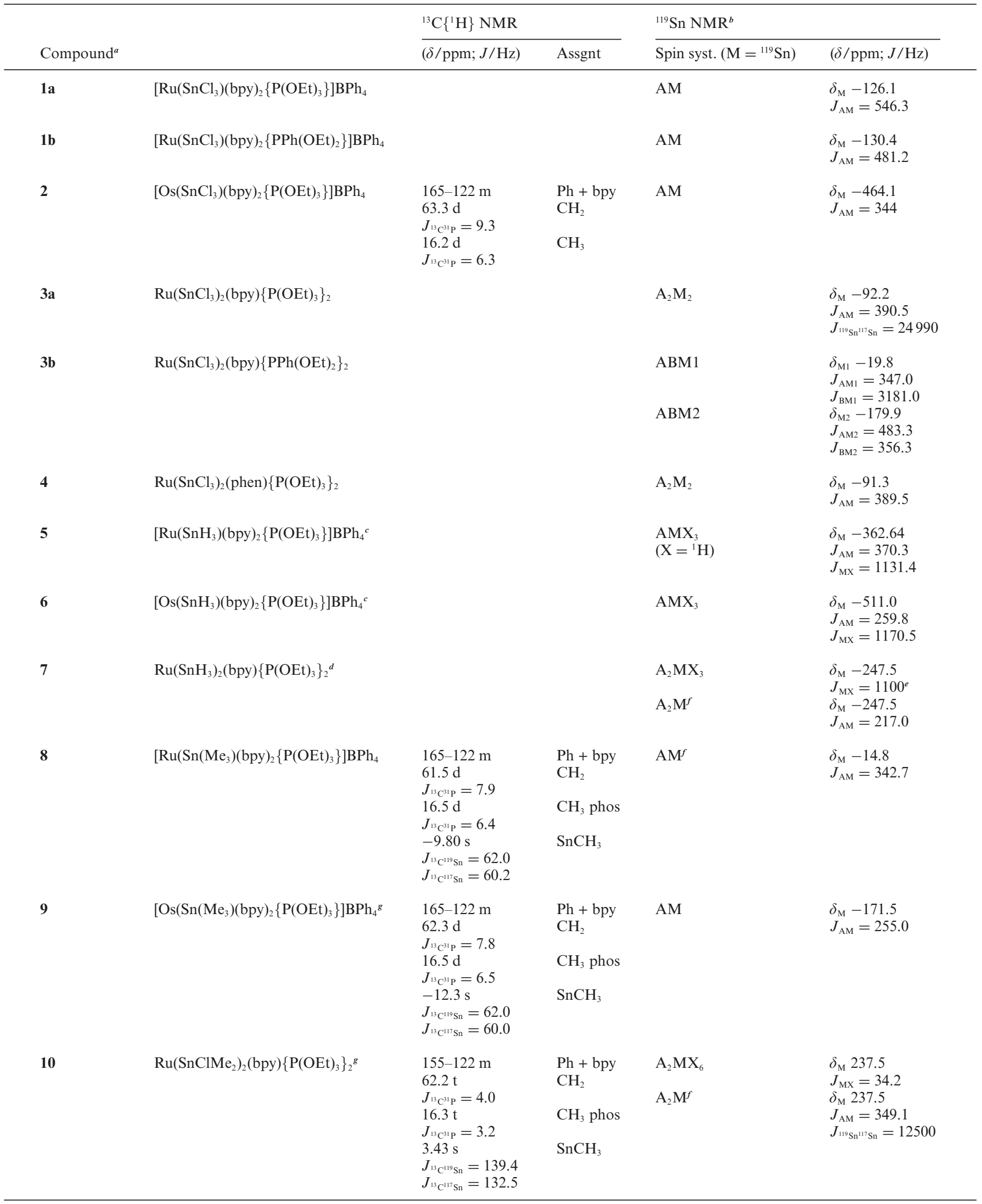


Table 2 (Contd.)

\begin{tabular}{|c|c|c|c|c|c|}
\hline \multirow[b]{2}{*}{ Compound $^{a}$} & & \multicolumn{2}{|l|}{${ }^{13} \mathrm{C}\left\{{ }^{1} \mathrm{H}\right\}$ NMR } & \multicolumn{2}{|l|}{${ }^{119} \mathrm{Sn} \mathrm{NMR}^{b}$} \\
\hline & & $(\delta / \mathrm{ppm} ; J / \mathrm{Hz})$ & Assgnt & Spin syst. $\left(\mathrm{M}={ }^{119} \mathrm{Sn}\right)$ & $(\delta / \mathrm{ppm} ; J / \mathrm{Hz})$ \\
\hline 11 & {$\left[\mathrm{Ru}\left\{\mathrm{Sn}(\mathrm{C} \equiv \mathrm{C} p \text {-tolyl })_{3}\right\}(\mathrm{bpy})_{2}\left\{\mathrm{P}(\mathrm{OEt})_{3}\right\}\right] \mathrm{BPh}_{4}$} & $\begin{array}{l}165-122 \mathrm{~m} \\
108.9 \mathrm{~s} \\
J^{13} \mathrm{C}^{119} \mathrm{Sn}=41 \\
93.5 \mathrm{~s} \\
J_{13} \mathrm{C}^{119} \mathrm{Sn}=207 \\
62.3 \mathrm{~d} \\
J^{13} \mathrm{C}^{31} \mathrm{P}=8.2 \\
21.58 \mathrm{~s} \\
16.3 \mathrm{~d} \\
J^{{ }^{13} \mathrm{C}^{31} \mathrm{P}}=5.8\end{array}$ & $\begin{array}{l}\mathrm{Ph}+\text { bpy } \\
\mathrm{C} \beta \\
\mathrm{C} \alpha \\
\mathrm{CH}_{2} \\
\mathrm{CH}_{3} \\
p \text {-tolyl } \\
\mathrm{CH}_{3} \text { phos }\end{array}$ & $\mathrm{AM}$ & $\begin{array}{l}\delta_{\mathrm{M}}-288.5 \\
J_{\mathrm{AM}}=459.7\end{array}$ \\
\hline 12 & {$\left[\mathrm{Ru}\left\{\mathrm{Sn}\left(\mathrm{C} \equiv \mathrm{CBu}^{t}\right)_{3}\right\}(\mathrm{bpy})_{2}\left\{\mathrm{P}(\mathrm{OEt})_{3}\right\}\right] \mathrm{BPh}_{4}{ }^{g}$} & $\begin{array}{l}165-122 \mathrm{~m} \\
116.7 \mathrm{~s} \\
J_{{ }^{13} \mathrm{C}^{119} \mathrm{Sn}}=43 \\
83.1 \mathrm{~s} \\
J_{{ }^{13} \mathrm{C}^{119} \mathrm{Sn}}=200 \\
62.3 \mathrm{~d} \\
J^{1{ }^{3}} \mathrm{C}^{31} \mathrm{P}=8.6 \\
28.7 \mathrm{~s} \\
31.9 \mathrm{~s} \\
16.4 \mathrm{~d} \\
J_{{ }^{13} \mathrm{C}^{31} \mathrm{P}}=6.2\end{array}$ & $\begin{array}{l}\mathrm{Ph}+\text { bpy } \\
\mathrm{C} \beta \\
\mathrm{C} \alpha \\
\mathrm{CH}_{2} \\
\mathrm{C}\left(\mathrm{CH}_{3}\right)_{3} \\
\mathrm{C}\left(\mathrm{CH}_{3}\right)_{3} \\
\mathrm{CH}_{3} \text { phos }\end{array}$ & $\mathrm{AM}$ & $\begin{array}{l}\delta_{\mathrm{M}}-288.5 \\
J_{\mathrm{AM}}=448.6\end{array}$ \\
\hline 13 & {$\left[\mathrm{Os}\left\{\mathrm{Sn}(\mathrm{C} \equiv \mathrm{C} p \text {-tolyl })_{3}\right\}(\mathrm{bpy})_{2}\left\{\mathrm{P}(\mathrm{OEt})_{3}\right\}\right] \mathrm{BPh}_{4}$} & $\begin{array}{l}165-122 \mathrm{~m} \\
108.1 \mathrm{~s} \\
J^{13} \mathrm{C}^{119} \mathrm{Sn}=59 \\
93.0 \mathrm{~s} \\
J^{13} \mathrm{C}^{119} \mathrm{Sn}=227 \\
J^{13} \mathrm{C}^{117} \mathrm{Sn}=216 \\
62.3 \mathrm{~d} \\
J^{13} \mathrm{C}^{31 \mathrm{P}}=8.6 \\
21.3 \mathrm{~s} \\
16.3 \mathrm{~d} \\
J^{13} \mathrm{C}^{31 \mathrm{P}}=6.3\end{array}$ & $\begin{array}{l}\mathrm{Ph}+\text { bpy } \\
\mathrm{C} \beta \\
\mathrm{C} \alpha \\
\mathrm{CH}_{2} \\
\mathrm{CH}_{3} \\
p \text {-tolyl } \\
\mathrm{CH}_{3} \text { phos }\end{array}$ & $\mathrm{AM}$ & $\begin{array}{l}\delta_{\mathrm{M}}-463.3 \\
J_{\mathrm{AM}}=315.5\end{array}$ \\
\hline 14 & $\mathrm{Ru}\left\{\mathrm{Sn}(\mathrm{C} \equiv \mathrm{C} p \text {-tolyl })_{3}\right\}_{2}($ bpy $)\left\{\mathrm{P}(\mathrm{OEt})_{3}\right\}_{2}{ }^{g}$ & 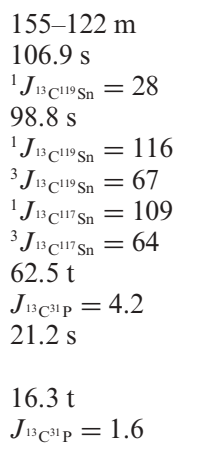 & $\begin{array}{l}\mathrm{Ph}+\text { bpy } \\
\mathrm{C} \beta \\
\mathrm{C} \alpha \\
\mathrm{CH}_{2} \\
\mathrm{CH}_{3} \\
p \text {-tolyl } \\
\mathrm{CH}_{3} \text { phos }\end{array}$ & $\mathrm{A}_{2} \mathrm{M}$ & 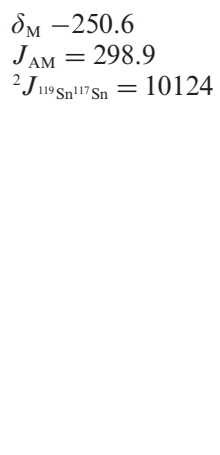 \\
\hline 15 & $\mathrm{Ru}\left\{\mathrm{Sn}\left(\mathrm{C} \equiv \mathrm{CBu}^{t}\right)_{3}\right\}_{2}(\mathrm{bpy})\left\{\mathrm{P}(\mathrm{OEt})_{3}\right\}_{2}{ }^{g}$ & $\begin{array}{l}155-122 \mathrm{~m} \\
113.6 \mathrm{~s} \\
J^{{ }^{13}} \mathrm{C}^{119} \mathrm{Sn}=25 \\
87.2 \mathrm{~s} \\
{ }^{1} J^{13} \mathrm{C}^{119} \mathrm{Sn}=143 \\
{ }^{3} J_{{ }^{13}} \mathrm{C}^{119} \mathrm{Sn}=64 \\
{ }^{1} J^{13} \mathrm{C}^{117} \mathrm{Sn}=138 \\
{ }^{3} J^{13} \mathrm{C}^{117} \mathrm{Sn}=61 \\
61.9 \mathrm{t} \\
J^{13} \mathrm{C}^{311} \mathrm{P}=4.2 \\
31.6 \mathrm{~s} \\
30.7 \mathrm{~s} \\
16.4 \mathrm{t} \\
J^{13} \mathrm{C}^{31} \mathrm{P}=3.6\end{array}$ & $\begin{array}{l}\mathrm{Ph}+\text { bpy } \\
\mathrm{C} \beta \\
\mathrm{C} \alpha \\
\\
\mathrm{CH}_{2} \\
\mathrm{CH}_{3} \text { phos } \\
\mathrm{C}\left(\mathrm{CH}_{3}\right)_{3} \\
\mathrm{C}\left(\mathrm{CH}_{3}\right)_{3}\end{array}$ & $\mathrm{~A}_{2} \mathrm{M}$ & $\begin{array}{l}\delta_{\mathrm{M}}-251.5 \\
J_{\mathrm{AM}}=292.5 \\
J_{{ }^{119} \mathrm{Sn}^{117} \mathrm{Sn}}=10065\end{array}$ \\
\hline
\end{tabular}


Table 2 (Contd.)

\begin{tabular}{|c|c|c|c|c|c|}
\hline Compound $^{a}$ & & \multicolumn{2}{|l|}{${ }^{13} \mathrm{C}\left\{{ }^{1} \mathrm{H}\right\} \mathrm{NMR}$} & \multicolumn{2}{|l|}{${ }^{119} \mathrm{Sn} \mathrm{NMR}^{b}$} \\
\hline 16 & $\mathrm{Ru}\left\{\mathrm{Sn}(\mathrm{C} \equiv \mathrm{C} p \text {-tolyl })_{3}\right\}_{2}($ phen $)\left\{\mathrm{P}(\mathrm{OEt})_{3}\right\}_{2}{ }^{g}$ & 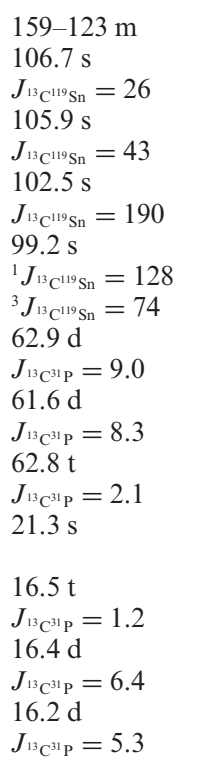 & $\begin{array}{l}\mathrm{Ph}+\text { phen } \\
\mathrm{C} \beta \\
\mathrm{C} \alpha \\
\mathrm{CH}_{2} \\
\\
\mathrm{CH}_{3} \\
p \text {-tolyl } \\
\mathrm{CH}_{3} \text { phos }\end{array}$ & $\begin{array}{l}\mathrm{A}_{2} \mathrm{M} \\
\mathrm{ABM} 1 \\
\mathrm{ABM} 2\end{array}$ & $\begin{array}{l}\delta_{\mathrm{M}}-246.2 \\
J_{\mathrm{AM}}=297.0 \\
{ }^{2} J_{{ }^{11} \mathrm{~S}^{117} \mathrm{Sn}}=10030 \\
\delta_{\mathrm{M} 1}-246.2 \\
J_{\mathrm{AM} 1}=349.7 \\
J_{\mathrm{BM} 1}=2604.9 \\
\delta_{\mathrm{M} 2}-311.5 \\
J_{\mathrm{AM} 2}=491.8 \\
J_{\mathrm{BM} 2}=308.0\end{array}$ \\
\hline 17 & $\mathrm{Ru}\left\{\mathrm{Sn}\left(\mathrm{C} \equiv \mathrm{CBu}^{t}\right)_{3}\right\}_{2}(\mathrm{phen})\left\{\mathrm{P}(\mathrm{OEt})_{3}\right\}_{2}{ }^{g}$ & $\begin{array}{l}155.7-124.7 \mathrm{~m} \\
113.4 \mathrm{~s} \\
{ }^{1} J^{13} \mathrm{C}^{119} \mathrm{Sn}=25.8 \\
87.3 \mathrm{~s} \\
{ }^{1} J^{13} \mathrm{C}^{119} \mathrm{Sn}=145 \\
{ }^{3} J_{{ }^{13}} \mathrm{C}^{119} \mathrm{Sn}=64 \\
{ }^{1} J^{13} \mathrm{C}^{117} \mathrm{Sn}=137 \\
{ }^{3} J^{13} \mathrm{C}^{117} \mathrm{Sn}=61 \\
61.9 \mathrm{t} \\
J^{13} \mathrm{C}^{311} \mathrm{P}=4.2 \\
31.8 \mathrm{~s} \\
31.3 \mathrm{~s} \\
16.4 \mathrm{t} \\
J^{13} \mathrm{C}^{31 \mathrm{P}}=3.6\end{array}$ & $\begin{array}{l} \\
\mathrm{CH}_{2} \\
\mathrm{C}\left(\mathrm{CH}_{3}\right)_{3} \\
\mathrm{C}\left(\mathrm{CH}_{3}\right)_{3} \\
\mathrm{CH}_{3} \text { phos }\end{array}$ & $\mathrm{A}_{2} \mathrm{M}$ & $\begin{array}{l}\delta_{\mathrm{M}}-247.5 \\
J_{\mathrm{AM}}=290.3 \\
{ }^{2} J_{119} \mathrm{Sn}^{117} \mathrm{Sn} \\
=9335\end{array}$ \\
\hline
\end{tabular}

${ }^{a} \mathrm{In} \mathrm{CD}_{2} \mathrm{Cl}_{2}$ at $20{ }^{\circ} \mathrm{C}$, unless otherwise noted. ${ }^{b}$ From external ${ }^{119} \mathrm{SnMe}_{4} \cdot{ }^{c}$ In thf- $\mathrm{d}^{8} .{ }^{d} \mathrm{In}$ benzene- $\mathrm{d}^{6} .{ }^{e}$ Estimated values due to the poor quality of the proton-coupled spectrum. ${ }^{f 119} \mathrm{Sn}\left\{{ }^{1} \mathrm{H}\right\}$ NMR. ${ }^{g} \mathrm{In}$ acetone-d $\mathrm{d}^{6}$.

unambiguously assign a geometry in solution to 7 , i.e., to decide between cis-cis or cis-trans geometry although, by analogy with the trichlorostannyl precursor $\mathbf{3 a}$, cis-trans geometry of type $\mathbf{V}$ is tentatively proposed.

\section{Characterisation of organostannyl complexes $[\mathrm{M}]-\mathrm{SnMe}_{3}$ and $[\mathrm{M}]-\mathrm{Sn}(\mathrm{C} \equiv \mathrm{CR})_{3}$}

The trimethylstannyl $\left[\mathrm{M}\left(\mathrm{SnMe}_{3}\right)(\mathrm{bpy})_{2}\left\{\mathrm{P}(\mathrm{OEt})_{3}\right\}\right] \mathrm{BPh}_{4} \quad$ complexes are stable red (8) or black (9) solids, which were characterised by analytical and spectroscopic (IR, ${ }^{1} \mathrm{H},{ }^{31} \mathrm{P},{ }^{13} \mathrm{C}$ and ${ }^{119} \mathrm{Sn}$ NMR) data and by X-ray crystal structure determination of $\mathbf{8}$.

The asymmetric unit contains a tetraphenylborate anion, a ruthenium complex cation and an acetone solvent molecule. The geometrical parameters of both solvent molecule and anion are as expected and do not require further comment. The cation complex (Fig. 1) consists of a ruthenium atom coordinated by four nitrogen atoms from two bidentate 2,2'-bipyridines, a phosphorus atom of a triethoxyphosphite ligand, and a tin atom from a trimethylstannyl ligand. The coordination polyhedron is a slightly distorted octahedron, in which the tin and the phosphorus atom are mutually cis. The other positions are occupied by nitrogen atoms. The 2,2'-bipyridine ligands are usually anisobidentate ${ }^{20}$ and, in this case, the shorter $\mathrm{Ru}-\mathrm{N}$ distances are those with another trans nitrogen atom. The $\mathrm{Ru}-\mathrm{N}$ distance trans to the tin atom is about $0.4 \AA$ longer than that trans to a phosphorus atom (Table 3 ). The $\mathrm{Ru}-\mathrm{Sn}$ distance, 2.6521(7) $\AA$, is similar to values previously reported, ${ }^{1 b, 21}$ but the $\mathrm{Ru}-\mathrm{P}$ distance, 2.210 (2) $\AA$, is slightly shorter than those previously published. ${ }^{7 a, 22}$

The main source of the distortion is probably the small bite of the two bidentate ligands, with chelate angles averaging $77.3(2)^{\circ}$, and the axial angles show some deviation from expected values. It is noteworthy that the value of the $\mathrm{P}-\mathrm{Ru}-\mathrm{N}$ angle, $169.5(1)^{\circ}$, shows divergence from the expected regularity. The phosphorus atom is also clearly out of the plane defined by the trans 2,2 bipyridine ligand, by $0.551(9) \AA$. This is probably due to the steric requirements of the phosphite ligand, and contrasts with the parameters found for the other monodentate ligand, since the 
Table 3 Bond lengths $(\AA)$ and angles $\left(^{\circ}\right)$ for $\mathbf{8}$

\begin{tabular}{lcll}
\hline $\mathrm{Ru}-\mathrm{N}(21)$ & $2.062(5)$ & $\mathrm{Ru}-\mathrm{N}(31)$ & $2.085(5)$ \\
$\mathrm{Ru}-\mathrm{N}(22)$ & $2.114(4)$ & $\mathrm{Ru}-\mathrm{N}(32)$ & $2.157(5)$ \\
$\mathrm{Ru}-\mathrm{P}(1)$ & $2.2101(17)$ & $\mathrm{Ru}-\mathrm{Sn}$ & $2.6521(7)$ \\
$\mathrm{Sn}-\mathrm{C}(3)$ & $2.171(7)$ & $\mathrm{Sn}-\mathrm{C}(2)$ & $2.192(7)$ \\
$\mathrm{Sn}-\mathrm{C}(1)$ & $2.198(5)$ & & \\
& & & \\
$\mathrm{N}(21)-\mathrm{Ru}-\mathrm{N}(31)$ & $167.81(19)$ & $\mathrm{N}(21)-\mathrm{Ru}-\mathrm{N}(22)$ & $77.38(19)$ \\
$\mathrm{N}(31)-\mathrm{Ru}-\mathrm{N}(22)$ & $94.73(18)$ & $\mathrm{N}(21)-\mathrm{Ru}-\mathrm{N}(32)$ & $93.45(19)$ \\
$\mathrm{N}(31)-\mathrm{Ru}-\mathrm{N}(32)$ & $77.26(19)$ & $\mathrm{N}(22)-\mathrm{Ru}-\mathrm{N}(32)$ & $91.18(17)$ \\
$\mathrm{N}(21)-\mathrm{Ru}-\mathrm{P}(1)$ & $99.09(14)$ & $\mathrm{N}(31)-\mathrm{Ru}-\mathrm{P}(1)$ & $90.23(14)$ \\
$\mathrm{N}(22)-\mathrm{Ru}-\mathrm{P}(1)$ & $169.56(14)$ & $\mathrm{N}(32)-\mathrm{Ru}-\mathrm{P}(1)$ & $98.88(12)$ \\
$\mathrm{N}(21)-\mathrm{Ru}-\mathrm{Sn}$ & $88.02(13)$ & $\mathrm{N}(31)-\mathrm{Ru}-\mathrm{Sn}$ & $100.87(14)$ \\
$\mathrm{N}(22)-\mathrm{Ru}-\mathrm{Sn}$ & $86.31(13)$ & $\mathrm{N}(32)-\mathrm{Ru}-\mathrm{Sn}$ & $176.75(13)$ \\
$\mathrm{P}(1)-\mathrm{Ru}-\mathrm{Sn}$ & $83.73(5)$ & $\mathrm{C}(3)-\mathrm{Sn}-\mathrm{C}(2)$ & $103.8(3)$ \\
$\mathrm{C}(3)-\mathrm{Sn}-\mathrm{C}(1)$ & $103.0(3)$ & $\mathrm{C}(2)-\mathrm{Sn}-\mathrm{C}(1)$ & $102.5(3)$ \\
$\mathrm{C}(3)-\mathrm{Sn}-\mathrm{Ru}$ & $112.8(2)$ & $\mathrm{C}(2)-\mathrm{Sn}-\mathrm{Ru}$ & $120.37(19)$ \\
$\mathrm{C}(1)-\mathrm{Sn}-\mathrm{Ru}$ & $112.47(17)$ & & \\
& & & \\
\hline
\end{tabular}

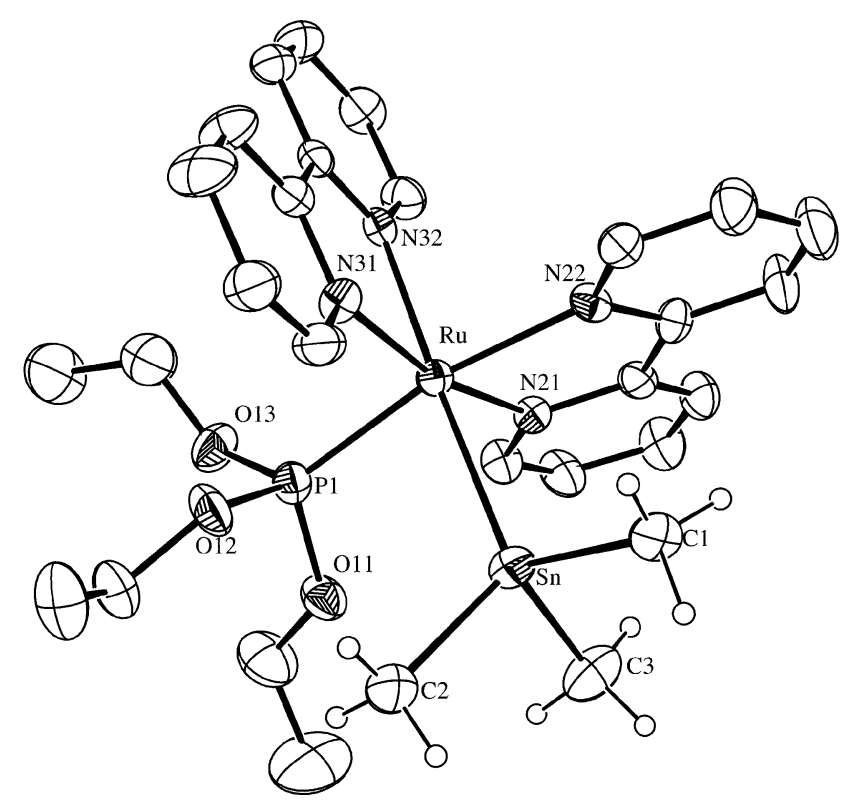

Fig. 1 ORTEP view of the cation $\left[\mathrm{Ru}\left(\mathrm{SnMe}_{3}\right)(\text { bpy })_{2}\left\{\mathrm{P}(\mathrm{OEt})_{3}\right\}\right]^{+} \boldsymbol{8}^{+}$drawn with thermal ellipsoids at $30 \%$ probability level.

$\mathrm{N}-\mathrm{Ru}-\mathrm{Sn}$ angle takes on a value of $176.75(13)^{\circ}$ (less than $4^{\circ}$ out of linearity) and the tin atom is only $0.081(9) \AA$ out of the plane defined by the bipyridine ligand in trans position.

Besides the signals of the bpy and $\mathrm{P}(\mathrm{OEt})_{3}$ ligands and $\mathrm{BPh}_{4}{ }^{-}$anion, the ${ }^{1} \mathrm{H}$ NMR spectra of trimethylstannyl complexes $\left[\mathrm{M}\left(\mathrm{SnMe}_{3}\right)(\mathrm{bpy})_{2}\left\{\mathrm{P}(\mathrm{OEt})_{3}\right\}\right] \mathrm{BPh}_{4} \mathbf{8}, 9$ showed a singlet near $-0.50 \mathrm{ppm}$, with the characteristic satellites of ${ }^{119} \mathrm{Sn}$ and ${ }^{117} \mathrm{Sn}$, attributed to the methyl protons of the $\mathrm{SnMe}_{3}$ ligand. In the ${ }^{13} \mathrm{C}$ spectra, the methyl signal of $\mathrm{SnMe}_{3}$ was identified at -9.80 (8) and -12.3 (9) ppm as a singlet, with the characteristic satellites due to coupling with the ${ }^{119} \mathrm{Sn}$ and ${ }^{117} \mathrm{Sn}$ nuclei. Support for this assignment came from HMQC experiments, which showed a correlation between this signal and the singlet near $-0.50 \mathrm{ppm}$ in the proton spectra, fitting the proposed formulation.

The proton-coupled ${ }^{119} \mathrm{Sn}$ NMR spectra confirmed the presence of the $\mathrm{SnMe}_{3}$ ligand, showing a complicated multiplet, due to coupling with the phosphorus nucleus of the $\mathrm{P}(\mathrm{OEt})_{3}$, and nine methyl protons. However, the ${ }^{1} \mathrm{H}$-decoupled spectra appeared as a doublet, fitting the proposed formulation for the complexes. In this case too, the values of the $J_{119} \mathrm{Sn}^{31} \mathrm{p}, 342.7-255.0 \mathrm{~Hz}$, suggest the mutually cis position of the phosphite and trimethylstannyl groups. On the basis of these data, a cis geometry VI, like that found in the solid state, is proposed for [M]-SnMe $\mathrm{S}_{3}$ complexes $\mathbf{8}$ and 9.

The ${ }^{1} \mathrm{H}$ and ${ }^{13} \mathrm{C}$ NMR spectra of bis(stannyl) complex $\mathrm{Ru}\left(\mathrm{SnClMe}_{2}\right)_{2}(\mathrm{bpy})\left[\mathrm{P}(\mathrm{OEt})_{3}\right]_{2} \mathbf{1 0}$ confirm the presence of the methylstannyl groups, showing a singlet at $0.29 \mathrm{ppm}$, with the characteristic satellites of the ${ }^{119} \mathrm{Sn}$ and ${ }^{117} \mathrm{Sn}$ nuclei in the proton spectra, and a singlet at $3.43 \mathrm{ppm}$ in the ${ }^{13} \mathrm{C}$ spectra, attributable to methyl $\mathrm{SnCH}_{3}$ resonances. A correlation in $\mathrm{HMQC}$ experiments between the proton signal at 0.29 and the ${ }^{13} \mathrm{C}$ signal at $3.43 \mathrm{ppm}$ was also observed. However, strong support for the presence of the bis(methyl) $\mathrm{SnClMe}_{2}$ ligand comes from the proton-coupled ${ }^{119} \mathrm{Sn}$ NMR spectra, which show a triplet of multiplets (seven signals each), due to coupling with two equivalent phosphorus nuclei of the phosphite and the six protons of the two methyl substituents. A computer simulation using an $\mathrm{A}_{2} \mathrm{MX}_{6}$ model $\left(\mathrm{M}={ }^{119} \mathrm{Sn}, \mathrm{X}=\right.$ ${ }^{1} \mathrm{H}, \mathrm{A}={ }^{31} \mathrm{P}$ ) gave a good fit between experimental and calculated spectra, supporting the presence of the $\mathrm{SnClMe}_{2}$ ligand. The value of $349.1 \mathrm{~Hz}$ for $J_{119} \mathrm{Sn}^{31} \mathrm{P}$ also suggests the mutually cis position of the phosphite and $\mathrm{SnCl}\left(\mathrm{CH}_{3}\right)_{2}$ groups. Lastly, the two $\mathrm{SnClMe}_{2}$ ligands are in a mutually trans position, fitting the high value ${ }^{19}$ $(12500 \mathrm{~Hz})$ observed for the $J_{119} \mathrm{Sn}^{117} \mathrm{Sn}$ of the two stannyl groups. On the basis of these data, a trans-cis geometry of type VII is reasonable for bis(methylstannyl) derivative $\mathbf{1 0}$.

Complexes containing one tris(alkynyl)stannyl group, of the type $\left[\mathrm{M}\left\{\mathrm{Sn}(\mathrm{C} \equiv \mathrm{CR})_{3}\right\}(\text { bpy })_{2}\left\{\mathrm{P}(\mathrm{OEt})_{3}\right\}\right] \mathrm{BPh}_{4}$, were obtained for both ruthenium $(\mathbf{1 1}, \mathbf{1 2})$ and osmium $(\mathbf{1 3})$ central metals, but bis(trialkynylstannyl) $\mathrm{Ru}\left[\mathrm{Sn}(\mathrm{C} \equiv \mathrm{CR})_{3}\right]_{2}(\mathrm{~N}-\mathrm{N})\left[\mathrm{P}(\mathrm{OEt})_{3}\right]_{2}$ derivatives (14-17) were only observed for ruthenium. All complexes were reddish-brown solids, stable in air and in solutions of organic solvents, where they behave as $1: 1$ electrolytes (11-13) or nonelectrolytes (14-17). Analytical and spectroscopic data (IR and NMR) support the proposed formulation.

The IR spectra of all the alkynylstannyl complexes 11-17 show a medium-intensity band at $2126-2115 \mathrm{~cm}^{-1}$, attributed to the $v_{\mathrm{C} \equiv \mathrm{C}}$ of the alkynyl group. Diagnostic for the presence of the $\mathrm{Sn}(\mathrm{C} \equiv \mathrm{CR})_{3}$ ligand were the ${ }^{13} \mathrm{C},{ }^{1} \mathrm{H}$ and ${ }^{119} \mathrm{Sn}$ NMR spectra. In the ${ }^{13} \mathrm{C}$ spectra, two singlets with the characteristic satellites, due to coupling with the ${ }^{117} \mathrm{Sn}$ and ${ }^{119} \mathrm{Sn}$ nuclei, were observed between 116 and $83 \mathrm{ppm}$ and attributed to the $\mathrm{C} \alpha$ and $\mathrm{C} \beta$ carbon resonances of the $\operatorname{Sn}(\mathrm{C} \alpha \equiv \mathrm{C} \beta \mathrm{R})_{3}$ group (Table 2). The values observed for $J_{13}{ }^{119} \mathrm{Sn}, 227-116 \mathrm{~Hz}$ in one case and $59-25 \mathrm{~Hz}$ in the other, clearly allow carbon resonances to be assigned. Both ${ }^{1} \mathrm{H}$ and ${ }^{13} \mathrm{C}$ NMR spectra of the alkynylstannyl complexes also showed the singlet attributed to methyl substituents of the $p$-tolylC $\equiv \mathrm{C}$ and $\mathrm{Bu}^{t} \mathrm{C} \equiv \mathrm{C}$ groups, fitting the presence of the $\mathrm{Sn}(\mathrm{C} \equiv \mathrm{CR})_{3}$ ligand.

In the temperature range between +20 and $-80{ }^{\circ} \mathrm{C}$, the ${ }^{31} \mathrm{P}$ NMR spectra of monostannyl complexes $\left[\mathrm{M}\left\{\mathrm{Sn}(\mathrm{C} \equiv \mathrm{CR})_{3}\right\}\right.$ (bpy) $\left.{ }_{2}\left\{\mathrm{P}(\mathrm{OEt})_{3}\right\}\right] \mathrm{BPh}_{4}$ 11-13 appeared as a sharp singlet, with the characteristic satellites of ${ }^{117} \mathrm{Sn}$ and ${ }^{119} \mathrm{Sn}$; the ${ }^{119} \mathrm{Sn}$ NMR spectra showed a doublet, due to coupling with the phosphorus of the phosphite. $J^{119} \mathrm{Sn}^{31} \mathrm{P}$ values fall in the range from 459.7 to $315.5 \mathrm{~Hz}$, suggesting the mutually cis position of the stannyl and phosphite ligands, as in type VIII geometry.

The ${ }^{119} \mathrm{Sn}$ NMR spectra of bis(trialkynylstannyl) complexes 14, 15 and 17 showed only one sharp triplet, indicating the 
magnetic equivalence of the two stannyl ligands. The spectra also showed the satellites due to ${ }^{119} \mathrm{Sn}-{ }^{117} \mathrm{Sn}$ coupling, and the value of about $10000 \mathrm{~Hz}$ suggests ${ }^{19}$ the mutually trans position of the two $\mathrm{Sn}(\mathrm{C} \equiv \mathrm{CR})_{3}$ ligands. In addition, in the temperature range between +20 and $-80{ }^{\circ} \mathrm{C}$, the ${ }^{31} \mathrm{P}$ spectra of the complexes appeared as a sharp singlet, with the characteristic satellites of ${ }^{117} \mathrm{Sn}$ and ${ }^{119} \mathrm{Sn}$, suggesting the magnetic equivalence of the two phosphite ligands. Lastly, the $J_{31^{1}{ }^{119} \mathrm{Sn}}$ value of about $300 \mathrm{~Hz}$ suggests ${ }^{18}$ the mutually cis position of the phosphite and stannyl ligands, fitting cis-trans geometry of type IX for bis(trialkynylstannyl) derivatives 14, 15 and 17.

Surprisingly, both ${ }^{119} \mathrm{Sn}$ and ${ }^{31} \mathrm{P}$ NMR spectra of the $\mathrm{Ru}\left[\mathrm{Sn}(\mathrm{C} \equiv \mathrm{C} p \text {-tolyl })_{3}\right]_{2}$ (phen) $\left[\mathrm{P}(\mathrm{OEt})_{3}\right]_{2}$ complex 16 showed two sets of signals, suggesting the presence of two isomers. The ${ }^{31} \mathrm{P}$ spectrum showed one singlet at $152.3 \mathrm{ppm}$ and an AB multiplet at 146.5-135.0 ppm, each pattern with the characteristic satellites of the ${ }^{117} \mathrm{Sn}$ and ${ }^{119} \mathrm{Sn}$ nuclei. The ${ }^{119} \mathrm{Sn}$ spectrum showed one triplet at $-246.2 \mathrm{ppm}$ and a complicated pattern (two multiplets) near $-280 \mathrm{ppm}$, which was simulated with two ABM spin systems (Table 2), fitting the presence of inequivalent stannyl groups. On the basis of these data, we propose the existence of two isomers with geometries of types IX and $\mathbf{X}$ (Scheme 5) for compound $\mathbf{1 6 .}$ In one isomer, the two magnetically equivalent stannyls are in a mutually trans position (IX), whereas in the other, both stannyls and phosphites are magnetically inequivalent and in a mutually cis position (X).

\section{Conclusions}

This report indicates that polypyridines (bpy and phen) as supporting ligands in ruthenium and osmium complexes allow the synthesis of a new series of mono- and bis(stannyl) derivatives. Among these, both mono- $\left[\mathrm{M}\left(\mathrm{SnH}_{3}\right)(\mathrm{bpy})_{2} \mathrm{P}\right] \mathrm{BPh}_{4}$ and the unprecedented bis(trihydridestannyl) $\mathrm{Ru}\left(\mathrm{SnH}_{3}\right)_{2}$ (bpy) $\mathrm{P}_{2}$ derivatives are interesting. An easy route for the synthesis of organostannyl derivatives $\left[\mathrm{M}\left(\mathrm{SnMe}_{3}\right)(\mathrm{bpy})_{2} \mathrm{P}\right] \mathrm{BPh}_{4}, \quad\left[\mathrm{Ru}\left\{\mathrm{Sn}(\mathrm{C} \equiv \mathrm{CR})_{3}\right\}(\text { bpy })_{2} \mathrm{P}\right] \mathrm{BPh}_{4}$ and $\mathrm{Ru}\left\{\mathrm{Sn}(\mathrm{C} \equiv \mathrm{CR})_{3}\right\}_{2}(\mathrm{~N}-\mathrm{N}) \mathrm{P}_{2}$ by substituting chloride in $[\mathrm{M}]-$ $\mathrm{SnCl}_{3}$ complexes with Grignard compounds $\mathrm{MgBrMe}$ or lithium acetylides is also reported.

\section{Acknowledgements}

We thank Daniela Baldan for technical assistance.

\section{References}

1 (a) K. M. Mackay and B. K. Nicholson, in Comprehensive Organometallic Chemistry, ed. G. Wilkinson, F. G. A. Stone and E. W. Abel, Pergamon Press, New York, USA, 1982, vol. 2, pp. 1043-1114; (b) M. S. Holt, W. L. Wilson and J. H. Nelson, Chem. Rev., 1989, 89, 1149; (c) A. G. Davies, in Comprehensive Organometallic Chemistry, ed. F. G. A. Stone, E. W. Abel and G. Wilkinson, Pergamon Press, New York, USA, 1995, vol. 2, pp. 218-297; (d) A. G. Davies, Organotin Chemistry, Wiley-VCH, Weinheim, Germany, 2004.

2 (a) U. Schubert and S. Grubert, Organometallics, 1996, 15, 4707-4713; (b) M. Akita, R. Hua, S. Nakanishi, M. Tanaka and Y. Moro-oka, Organometallics, 1997, 16, 5572-5584; (c) J.-P. Djukic, K. H. Dötz, M. Pfeffer, A. De, Cian and J. Fischer, Organometallics, 1997, 16, 51715182; (d) H. Nakazawa, Y. Yamaguchi, K. Kawamura and K. Miyoshi, Organometallics, 1997, 16, 4626-4635; (e) M. Baya, P. Crochet, M. A. Esteruelas, E. Gutierrez-Puebla and N. Ruiz, Organometallics, 1999, 18, 5034-5043; (f) C. E. F. Rickard, W. R. Roper, T. J. Woodman and L. J. Wright, Chem. Commun., 1999, 837-838; $(g)$ H. Adams, S. G.
Broughton, S. J. Walters and M. J. Winter, Chem. Commun., 1999, 1231-1232; (h) A. M. Clark, C. E. F. Rickard, W. R. Roper, T. J. Woodman and L. J. Wright, Organometallics, 2000, 19, 1766-1774; (i) S. Hermans and B. F. G. Johnson, Chem. Commun., 2000, 19551956; (j) N. R. Neale and T. D. Tilley, J. Am. Chem. Soc., 2002, 124, 3802-3803; (k) M. A. Esteruelas, A. Lledos, F. Maseras, M. Oliván, E. Oñate, M. A. Tajada and J. Tomàs, Organometallics, 2003, 22, $2087-$ 2096; (l) R. D. Adams, B. Captain, J. L. Smith, Jr., M. B. Hall, C. L. Beddie and C. E. Webster, Inorg. Chem., 2004, 43, 7576-7578; (m) N. R. Neale and T. D. Tilley, J. Am. Chem. Soc., 2005, 127, 14745-14755; (n) R. D. Adams, B. Captain, R. H. Herber, M. Johansson, I. Nowik, J. L. Smith and M. D. Smith, Inorg. Chem., 2005, 44, 6346-6358; (o) T. Sagawa, K. Ohtsuki, T. Ishiyama and F. Ozawa, Organometallics, 2005, 24, 1670-1677; (p) R. D. Adams, B. Captain, C. B. Hollandsworth, M. Johansson and J. L. Smith, Jr., Organometallics, 2006, 25, 3848-3855.

3 (a) H. C. Clark and B. K. Hunter, J. Organomet. Chem., 1971, 31, $227-$ 232; (b) J. R. Chipperfield, A. C. Hayter and D. E. Webster, J. Chem. Soc., Dalton Trans., 1977, 485-490; (c) J. C. Luong, R. A. Faltynek and M. S. Wrighton, J. Am. Chem. Soc., 1980, 102, 7892-7900; (d) B. T. Huie, S. W. Kirtley, C. B. Knobler and H. D. Kaesz, J. Organomet. Chem., 1981, 213, 45-62; (e) S. B. McCullen and T. L. Brown, J. Am. Chem. Soc., 1982, 104, 7496-7500; (f) S. P. Foster and K. M. Mackay, J. Organomet. Chem., 1983, 247, 21-26; (g) B. A. Narayanan and J. K. Kochi, Inorg. Chim. Acta, 1986, 122, 85-90; (h) D. E. Westerberg, B. E. Sutherland, J. C. Huffman and K. G. Caulton, J. Am. Chem. Soc., 1988, 110, 1642-1643; (i) G. F. P. Warnock, L. C. Moodie and J. E. Ellis, J. Am. Chem. Soc., 1989, 111, 2131-2141; (j) J. P. Bullock, M. C. Palazzotto and K. R. Mann, Inorg. Chem., 1990, 29, 4413-4421; (k) R. J. Sullivan and T. L. Brown, J. Am. Chem. Soc., 1991, 113, 9155-9161; (l) M. L. Loza and R. H. Crabtree, Inorg. Chim. Acta, 1995, 236, 63-66; (m) T. L. Utz, P. A. Leach, S. J. Geib and N. J. Cooper, Chem. Commun., 1997, 847-848; (n) B. Biswas, M. Sugimoto and S. Sakaki, Organometallics, 1999, 18, 4015-4026; (o) Y.-S. Chen and J. E. Ellis, Inorg. Chim. Acta, 2000, 300-302, 675-682; (p) D. Christendat, I. Wharf, A.-M. Lebuis, I. S. Butler and D. F. G. Gilson, Inorg. Chim. Acta, 2002, 329, 36-44; (q) T. Matsubara, Organometallics, 2003, 22, 4297-4304.

4 (a) J. N. Coupé, E. Jordão, M. A. Fraga and M. Mendes, J. Appl. Catal. A, 2000, 199, 45; (b) S. Hermans, R. Raja, J. M. Thomas, B. F. G. Johnson, G. Sankar and D. Gleeson, Angew. Chem., Int. Ed., 2001, 40, 1211; (c) G. W. Huber, J. W. Shabaker and J. A. Dumesic, Science, 2003, 300, 2075; (d) R. D. Adams, B. Captain, M. Johansson and J. L. Smith, Jr., J. Am. Chem. Soc., 2005, 127, 488-489.

5 (a) J. A. Weinstein, J. van Slageren, D. J. Stufkens, S. Zalis and M. W. George, J. Chem. Soc., Dalton Trans., 2001, 2587-2592; (b) M. Turki, C. Daniel, S. Zalis, A. Vlcek, Jr., J. van Slageren and D. J. Stufkens, J. Am. Chem. Soc., 2001, 123, 11431-11440; (c) J. van Slageren and D. J. Stufkens, Inorg. Chem., 2001, 40, 277-285; (d) M. P. Aarnts, M. P. Wilms, K. Peelen, J. Fraanje, K. Goubitz, F. Hartl, D. J. Stufkens, E. J. Baerends and A. Vlcek, Jr., Inorg. Chem., 1996, 35, 5468-5477; (e) M. P. Aarnts, D. J. Stufkens, M. P. Wilms, E. J. Baerends, A. Vlcek, Jr., I. P. Clark, M. W. George and J. J. Turner, Chem.-Eur. J., 1996, 2, 1556.

6 (a) G. Albertin, S. Antoniutti and M. Bortoluzzi, Inorg. Chem., 2004, 43, 1328-1335; (b) G. Albertin, S. Antoniutti, A. Bacchi, C. D'Este and G. Pelizzi, Inorg. Chem., 2004, 43, 1336-1349; (c) G. Albertin, S. Antoniutti and S. Pizzol, J. Organomet. Chem., 2004, 689, 1639-1647.

7 (a) G. Albertin, S. Antoniutti, M. Bortoluzzi, J. Castro-Fojo and S. García-Fontán, Inorg. Chem., 2004, 43, 4511-4522; (b) G. Albertin, S. Antoniutti, M. Bortoluzzi and G. Zanardo, J. Organomet. Chem., 2005, 690, 1726-1738.

8 (a) G. Albertin, S. Antoniutti, A. Bacchi, M. Bortoluzzi, G. Pelizzi and G. Zanardo, Organometallics, 2006, 25, 4235-4237; (b) G. Albertin, S. Antoniutti, A. Bacchi, G. Pelizzi and G. Zanardo, manuscript in preparation.

9 R. Rabinowitz and J. Pellon, J. Org. Chem., 1961, 26, 4623-4626.

10 G. Balacco, J. Chem. Inf. Comput. Sci., 1994, 34, 1235-1241; http://www.inmr.net/.

11 G. M. Sheldrick, SADABS. An empirical absorption correction program for area detector data, University of Göttingen, Germany, 1996.

12 P. McArdle, J. Appl. Crystallogr., 1995, 28, 65.

13 G. M. Sheldrick, SHELX-97. Program for the solution and refinement of crystal structures, University of Göttingen, Germany, 1997.

14 International Tables for X-ray Crystallography, vol. C, Kluwer, Dordrecht, 1992.

15 (a) A. Albinati, P. S. Pregosin and H. Rüegger, Inorg. Chem., 1984, 23, 3223-3229; (b) J. E. Ellis, D. W. Blackburn, P. Yuen and M. Jang, 
J. Am. Chem. Soc., 1993, 115, 11616-11617; (c) M. A. Esteruelas, F. J. Lahoz, M. Oliván, E. Oñate and L. A. Oro, Organometallics, 1994, 13, 4246-4257; (d) M. Weidenbruch, A. Stilter, W. Saak, K. Peters and H. G. von Schnering, J. Organomet. Chem., 1998, 560, 125-129; (e) T. Szymánska-Buzar and T. Glowiak, J. Organomet. Chem., 1999, 575, 98-107; (f) J. M. Allen, W. W. Brennessel, C. E. Buss, J. E. Ellis, M. E. Minyaev, M. Pink, G. F. Warnock, M. L. Winzenburg and V. G. Young, Jr., Inorg. Chem., 2001, 40, 5279-5284; (g) M. Lutz, B. Findeis, M. Haukka, T. A. Pakkanen and L. H. Gade, Eur. J. Inorg. Chem., 2001, 3155; (h) A. Fischer and O. F. Wendt, J. Chem. Soc., Dalton Trans., 2001, 1266; (i) M. A. Esteruelas, A. Lledós, O. Maresca, M. Oliván, E. Oñate and M. A. Tajada, Organometallics, 2004, 23, 14531456.

16 G. Albertin, S. Antoniutti, J. Castro, S. García-Fontán and G. Zanardo, Organometallics, 2007, 26, 2918-2930.

17 W. J. Geary, Coord. Chem. Rev., 1971, 7, 81-122.

18 (a) R. F. Bryan, Chem. Commun. (London), 1967, 355; (b) M. M. Möhlen, C. E. F. Rickard, W. R. Roper, G. R. Whittell and L. J. Wright, J. Organomet. Chem., 2006, 691, 4065-4075.
19 (a) L. J. Farrugia, B. R. James, C. R. Lassigne and E. W. Wells, Inorg. Chim. Acta, 1981, 53, L261; (b) L. J. Farrugia, B. R. James, C. R. Lassigne and E. W. Wells, Can. J. Chem., 1982, 60, 1304; (c) H. Moriyama, T. Aoki, S. Shinoda and Y. Saito, J. Chem. Soc., Chem. Commun., 1982, 500; (d) H. Moriyama, P. S. Pregosin, Y. Saito and T. Yamakawa, J. Chem. Soc., Dalton Trans., 1984, 2329.

20 See, for example: (a) R. Haid, R. Gutmann, T. Stampfl, C. Langes, G. Czermak, H. Kopacka, K.-H. Ongania and P. Brüggeller, Inorg. Chem., 2001, 40, 7099-7104; (b) C. W. Rogers, Y. Zhang, B. O. Patrick, W. E. Jones and M. O. Wolf, Inorg. Chem., 2002, 41, 1162-1169.

21 (a) G. R. Clark, K. R. Flower, W. R. Roper and L. J. Wright, Organometallics, 1993, 12, 259-260; (b) K. Kawamura, H. Nakazawa and K. Miyoshi, Organometallics, 1999, 18, 4785-4794.

22 (a) G. Albertin, S. Antoniutti, M. Bedin, J. Castro and S. GarcíaFontán, Inorg. Chem., 2006, 45, 3816-3825; (b) J. M. Butler, G. M. Gray and J. P. Claude, Polyhedron, 2004, 23, 1719-1729; (c) R. M. Carlos, D. R. Cardoso, E. E. Castellano, R. Z. Osti, A. J. Camargo, L. G. Macedo and D. W. Franco, J. Am. Chem. Soc., 2004, 126, 25462555. 